\author{
M. Desmurget · D. Pélisson · J.S. Grethe \\ G.E. Alexander $\cdot$ C. Urquizar $\cdot$ C. Prablanc \\ S.T. Grafton
}

\title{
Functional adaptation of reactive saccades in humans: a PET study
}

Received: 9 September 1999 / Accepted: 27 December 1999 / Published online: 25 March 2000

(C) Springer-Verlag 2000

\begin{abstract}
It is known that the saccadic system shows adaptive changes when the command sent to the extraocular muscles is inappropriate. Despite an abundance of supportive psychophysical investigations, the neurophysiological substrate of this process is still debated. The present study addresses this issue using $\mathrm{H}_{2}{ }^{15} \mathrm{O}$ positron emission tomography (PET). We contrasted three conditions in which healthy human subjects were required to perform saccadic eye movements toward peripheral visual targets. Two conditions involved a modification of the target location during the course of the initial saccade, when there is suppression of visual perception. In the RAND condition, intra-saccadic target displacement was random from trial-to-trial, precluding any systematic modification of the primary saccade amplitude. In the ADAPT condition, intra-saccadic target displacement was uniform, causing adaptive modification of the primary saccade amplitude. In the third condition (stationary, STAT), the target remained at the same location during the entire trial. Difference images reflecting regional cerebral-blood-flow changes attributable to the process of saccadic adaptation (ADAPT minus RAND; ADAPT minus STAT) showed a selective activation in the oculomotor cerebellar vermis (OCV; lobules VI and VII). This finding is consistent with neurophysiological studies in monkeys. Additional analyses indicated that the cerebellar activation was not related to kinematic factors, and
\end{abstract}

M. Desmurget · J.S. Grethe · G.E. Alexander · S.T. Grafton Department of Neurology, Emory University, Atlanta, GA 30322, USA

S.T. Grafton (

Department of Radiology and the PET imaging Center, Emory University, Atlanta, GA 30322, USA

M. Desmurget - D. Pélisson - C. Urquizar · C. Prablanc INSERM U534, Espace et Action, 16 av du Doyen Lépine, 69500 Bron, France

Current address: Scott T. Grafton,

Center for Cognitive Neuroscience, 6162 Moore Hall,

Dartmouth College, Hanover, NH 03755, USA,

e-mail: Scott.T.Grafton@ dartmouth.edu,

Tel.: +1-603-6460038, Fax: +1-603-6461181 that the absence of significant activation within the frontal eye fields (FEF) or the superior colliculus (SC) did not represent a false negative inference. Besides the contribution of the OCV to saccadic adaptation, we also observed, in the RAND condition, that the saccade amplitude was significantly larger when the previous trial involved a forward jump than when the previous trial involved a backward jump. This observation indicates that saccade accuracy is constantly monitored on a trial-totrial basis. Behavioral measurements and PET observations (RAND minus STAT) suggest that this single-trial control of saccade amplitude may be functionally distinct from the process of saccadic adaptation.

Key words Saccadic adaptation - Saccade - Adaptation . PET $\cdot$ Cerebellum $\cdot$ FEF $\cdot$ Colliculus

\section{Introduction}

Saccades are fast eye movements that shift the point of gaze from one position to another. Although they ordinarily occur too rapidly to be influenced by peripheral feedback loops, they exhibit a remarkable accuracy, suggesting that the sensory and motor components of the oculomotor system are precisely calibrated with each other. Throughout life, the adequacy of this calibration is constantly challenged by neural death, brain lesions, and biomechanical changes in the characteristics of the oculomotor apparatus. This indicates that saccade precision can only be maintained through an adaptive process, which continually updates the relation between the required displacement of the eyes and the oculomotor command. The existence of such a process was clearly demonstrated in patients presenting a monocular paresis of the extraocular muscles (Kommerell et al. 1976; Optican et al. 1985). In these patients, the normal yoking of the eyes is disrupted: while the unaffected eye exhibits normal saccadic responses, the deficient eye dramatically undershoots the target. When the unaffected eye is patched, forcing the subject to view only through the de- 
ficient eye, saccades gradually increase in size until movement accuracy is restored, which occurs after a few days of exposure. In accordance with Hering's law of equal innervation, this adaptive increase in size is observed for the "normal“ eye as well, leading that eye to overshoot the target when the patch is removed. Within a few days of practice without the patch, this initial imprecision disappears due to a progressive decrease of the saccadic gain, which progressively returns to its original value (de-adaptation).

Two main experimental approaches have been used to investigate saccadic adaptation (SA). The first approach, employed in monkeys, consists of surgically lesioning the extraocular muscles (Optican and Robinson 1980; Scudder et al. 1998). The second approach, used in both humans and monkeys, consists of changing the target location during the initial saccadic displacement. This noninvasive procedure, designated the target-jump paradigm, was first introduced by McLaughlin (1967). It involves three steps. First, the subject looks at a fixation point. Second, a target is presented in the peripheral visual field triggering a saccade. Third, during the saccadic response, the target location is slightly modified. Because the perception of small target displacements is impaired during fast eye movements (Matin 1982), the subject is not aware of this jump. As a consequence, the experimentally induced post-saccadic error is interpreted by the saccadic system as a deficit of the oculomotor command. Systematically repeating the same target displacement from trial to trial leads to a reduction of the post-saccadic error, by way of a gradual and adaptive change in saccade amplitude. This paradigm has allowed an extensive investigation of the properties of SA in humans and animals. One of the main results obtained within the last decade is that SA is spatially selective, i.e., it only transfers to a restricted range of eye displacements centered around the adapted saccade. With respect to this point, it was shown that adaptation of saccades in one direction did not generalize to saccades performed in a perpendicular or opposite direction (Deubel et al. 1986; Frens and Van Opstal 1994). Furthermore, it was observed that, for a fixed saccade direction, adaptation of movements of a given amplitude did not parametrically generalize to movements of other amplitudes (Miller et al. 1981; Frens and Van Opstal 1994; Albano 1996; Straube et al. 1997). The only positive transfer was found for saccades of similar amplitude and direction, even when triggered from different orbital positions (Frens and Van Opstal 1994; Albano 1996; Wallman and Fuchs 1998).

Although oculomotor plasticity is well documented from a behavioral perspective, its neural substrate remains controversial. One of the obvious anatomical candidates that may underlie SA is the superior colliculus (SC). This structure is known to be critically involved in the generation of goal-directed saccades (for reviews, see Sparks and May 1990; Guitton 1991). Its deep layers (DLSC) contain saccade-related burst neurons (SRBNs), which are thought to encode the saccadic movement in a topographical motor map where the displacement is represented in polar coordinates (amplitude and direction). In DLSC, the SRBNs have a well-defined ,movement field", which means that they respond maximally for a given movement vector and that their firing rate decreases progressively as the movement diverges from the optimal vector. It was suggested that the actual saccadic displacement reflects the weighted output of the cell population recruited in the SC (Lee et al. 1988). This model fits well with the observation that SA only transfers to a range of displacements centered around the adapted saccade (Miller et al. 1981; Frens and Van Opstal 1994; Noto et al. 1999). Indeed, if oculomotor plasticity involves changes in the DLSC, the number of ,adapted cells" recruited is expected to decrease when the movement to be performed deviates from the adapted movement. A topographical organization similar to the one observed in the SC has been described in the frontal eye field (FEF; Bruce and Golberg 1985), suggesting that this cortical structure may also play a role in SA (Frens and Van Opstal 1994; Noto et al. 1999). Such a cortical contribution would be compatible with the observation that SA occurs quickly in humans, as compared with monkeys (see below).

The hypothesis that the SC represents the anatomical substrate for oculomotor plasticity was tested by recording unit activity of SRBNs in the DLSC or by electrically stimulating DLSC, in monkeys, after a target-jump paradigm was performed to induce SA. It was shown that the amplitude of saccades evoked by electrical stimulation of DLSC was not affected after eye movements had been adapted behaviorally (Fitzgibbon et al. 1986; Melis and van Gisbergen 1996). In order to account for this observation, it was initially proposed that SA occurred either upstream from the SC by affecting the signal entering this structure, or at the level of the SC itself by controlling the visual-to-motor transformation (Fitzgibbon et al. 1986). These interpretations were challenged by the subsequent demonstration that collicular motor cells showed the same pattern of discharge after saccadic adaptation even when the adapted saccade no longer terminated in the original movement field (Goldberg et al. 1993; Frens and Van Opstal 1997). This result is compatible with the idea that a corrective command is added to the collicular output signal downstream from the SC. The apparent contradiction between this conclusion and that initially derived from electrical stimulation studies (Fitzgibbon et al. 1986; Melis and van Gisbergen 1996) can be accounted for by assuming that the corrective command is added to the collicular output only under natural circumstances when the saccade is triggered by and directed toward a visual target. Additional evidence that an adaptive corrective command is added to the collicular output signal came from the finding that saccades induced by electrical stimulation of the SC could be adaptively modified with a target-jump paradigm (Melis and van Gisbergen 1996).

Obviously, a major anatomical candidate that may modulate the oculomotor command sent by the SC to the 
saccadic burst generator is the cerebellum. This structure is known to play an essential role in the generation of goal-directed saccades (Noda 1991; Lewis and Zee 1993). In particular, the complex formed by the fastigial nuclei and the oculomotor vermis (lobules VI and VII, Noda and Fujikado 1987) is thought to be critically involved in the control of saccade metrics (Ohtsuka and Noda 1991b; Fuchs et al. 1993; Robinson et al. 1993; Goffart and Pélisson 1998). The hypothesis that SA depends on cerebellar integrity has been supported by lesion studies indicating that patients suffering from syndromes associated with cerebellar dysfunction showed impaired SA capacities (Waespe and Baumgartner 1992). In addition to this point, it was also shown, in monkeys, that adaptive capabilities were dramatically impaired when the oculomotor cerebellar vermis (OCV) and/or the fastigial nuclei were lesioned (Optican and Robinson 1980; Goldberg et al. 1993; Takagi et al. 1998; Barash et al. 1999).

Experimental results questioning the hypothesis that SA relies exclusively on the collicular-cerebellar loop have been recently reported by Melis and van Gisbergen (1996). These authors used a target-jump paradigm to adaptively modify the amplitude of ocular saccades triggered by electrical stimulation of the SC. After adaptation, they observed only a partial transfer of these electrically elicited saccades to visually elicited saccades. A possible explanation for this result is that the electrically stimulated and naturally generated saccades either do not recruit the same neuronal pools or fail to recruit them with the appropriate temporal organization (Goldberg et al. 1993; Melis and van Gisbergen 1996). An alternative hypothesis, based on the existence of direct projections from both the SC and the FEF to the brainstem saccadic generator, proposes that these two structures contribute to the motor command driving the saccadic-burst generator. This idea was developed by Melis and van Gisbergen (1996), who proposed ,a scheme in which saccadic eye movements are generated by two parallel pathways, one via the FEF and another via the SC, whose crude commands are weighted to determine the motor error command to the burst generator. This crude motor error command can be adjusted by a corrective side loop via the cerebellum, which is activated by both FEF and SC crude commands" ( $p$ 1758). According to this scheme, only a limited transfer of adaptation is expected from saccades involving exclusively one parallel pathway (e.g., saccades evoked from electrical stimulation of the SC) to saccades resulting from the simultaneous activation of the two parallel pathways (e.g., visually elicited saccades).

As shown by the previous observations, the anatomical substrate of SA is still far from elucidated. The issue may appear even more opaque, considering that most of the neurophysiological data available in the literature were obtained in monkeys and that the behavioral characteristics of the adaptation produced by the target-jump paradigm are very different in humans and monkeys. In particular, substantial variations exist in how human and monkey SA transfers across paradigms. In humans, saccade amplitude modifications are generally circum- scribed to the adapted movement and there is no transfer from reactive to volitional saccades (Erkelens and Hulleman 1993; Deubel 1995) or from eye movements to head movements (Kröller et al. 1996). In monkeys, by contrast, the adapted movement transfers significantly to both the head (Phillips et al. 1997) and a large variety of saccades (Fuchs et al. 1996). Another major difference between humans and monkeys concerns the time course of the adaptation. While substantial adaptation requires only a few tens of trials in humans (Deubel et al. 1986; Frens and Van Opstal 1994), several hundreds of saccades are necessary in monkeys (Fuchs et al. 1996; Straube et al. 1997; Scudder et al. 1998). These major behavioral disparities between humans and monkeys suggest that these two species ,simply employ different mechanisms of adaptation to solve apparently identical problems of saccadic gain control" (Fuchs et al. 1996, p 2534). In particular, the much quicker adaptation observed in humans may suggest a greater contribution of the cortical FEF complex (Frens and Van Opstal 1994). Another explanation might be, however, that SA is highly dependent on the experimental design. For instance, it has been suggested that the time-course differences observed between humans and monkey could be related to the fact that the number of targets used to produce SA is generally higher in monkeys. As shown by several studies, SA is slower to develop when the number of targets increases (Miller et al. 1981; Scudder et al. 1998).

In an attempt to identify the anatomical substrate of SA in healthy humans, we recently carried out a $\mathrm{H}_{2}{ }^{15} \mathrm{O}$ positron emission tomography (PET) study (Desmurget et al. 1998). Two conditions were contrasted in which the subjects were required to perform saccadic eye movements toward a visual target that was displaced during the course of the initial saccade. In the ,random“ condition, the target jump varied systematically in direction and magnitude from trial-to-trial, preventing SA from occurring. In the ,adapt" condition, by contrast, the target jump was consistent within the whole scan, inducing a progressive modification of the saccade magnitude. Difference images reflecting metabolic changes attributable to SA showed a selective activation in the posterior cerebellar vermis. In this initial study, however, no a priori hypothesis was formulated about the possible areas that might be involved in SA. As a consequence, severe statistical constraints were applied to adjust for multiple comparisons, increasing the probability of false negative inference (i.e., of concluding that an activation was not significant when it was). The drastic correction applied to the raw data may explain our failure to identify significant SA-related activations in the FEF or the SC. In addition to this explanation, our inability to detect an activation within the FEF or the SC may have been related to the choice of a random condition as a reference task for the calculation of difference images. It is possible that intra-saccadic target jumps elicit some single-trial corrective process, as reported for arm movements during prism adaptation (Rossetti et al. 1993; Redding and Wallace 1996). According to this hypothesis, both the 
FEF and the SC may be involved in SA in such a way that they are activated after every trial for which an abnormal postsaccadic error is observed. If this is the case, regional cerebral-blood-flow ( $\mathrm{rCBF}$ ) variations in these structures would have been artificially „erased“ in our initial study due to the comparison of a systematic-jump condition with a random-jump condition.

The main aim of the present paper is both to address the previous reservations and to test for the existence of a potential single-trial error control process. To achieve these goals, we proceeded in three successive steps. First, we re-analyzed our original data by focusing on the structures that have been reported in the literature as potential loci for SA, namely the FEF, the SC, and the OCV. This a priori prediction about the areas that may be activated during the process of SA allowed us to use more powerful statistical tests, significantly decreasing the probability of false negative inferences. Second, we analyzed the metrics of the saccades performed under the „random" condition in order to identify potential trial-totrial changes in saccade amplitude. Third, we contrasted the ,adapt" and ,random“ conditions with a third condition in which the target location was maintained stationary during the entire trial.

\section{Materials and methods}

Subjects and apparatus

Nine right-handed naive subjects (seven males, two females), from 22 to 59 years of age (mean: 30 years; SD: 12), participated in the study after informed consent was obtained. During the experiment, they were supine with their head immobilized in the scanner. The experimental apparatus is schematized in Fig. 1A. It consisted of a line of red light-emitting diodes positioned on a half-
Fig. 1. Schematic representation of the experimental apparatus $(\mathbf{A})$ and the experimental protocol (B). Time values obtained from a single trial are provided as an illustration of the actual timing of the oculomotor sequence. PET Positron emission tomography, $E O G$ electro-oculography
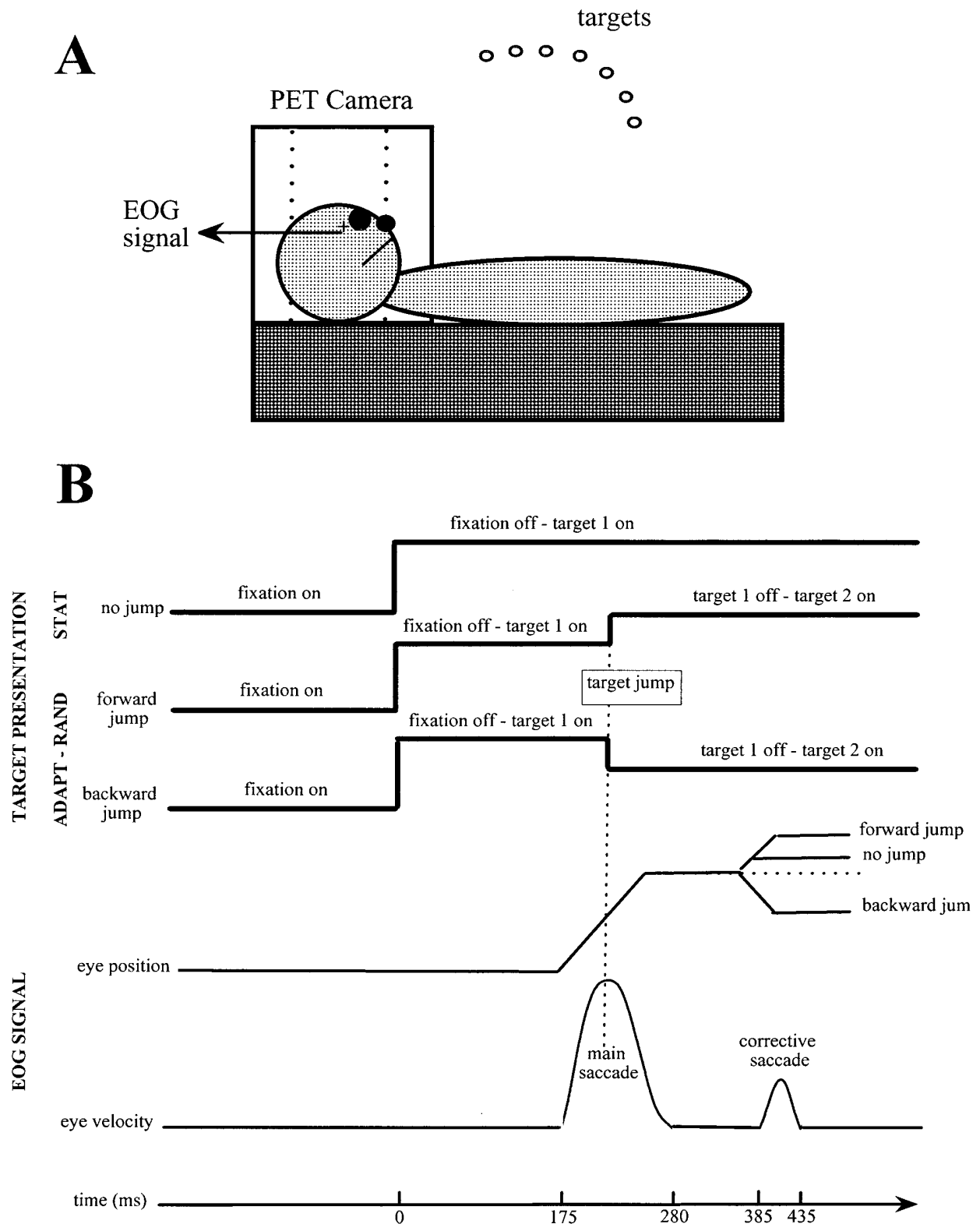
circle (radius $55 \mathrm{~cm}$ ). This circle was centered on the subjects' cyclopean eye (middle of the segment joining the two eyeballs) and adjusted to be in the same plane as the subjects' line of sight. The diodes were located every $2.5^{\circ}$ from $-20^{\circ}$ (left) to $32.5^{\circ}$ (right). Eye position was recorded binocularly by a DC electro-oculographic technique (EOG).

\section{Experimental conditions}

The protocol was approved by the institutional Human Investigation Committee of Emory University. Three experimental conditions were considered and repeated four times. Each of these conditions involved the same four steps (Fig. 1B): (1) one of three possible fixation points $\left(-20^{\circ},-10^{\circ}, 0^{\circ}\right)$ was turned on for $1.5 \mathrm{~s}$; (2) the initial fixation point was turned off while the target was simultaneously turned on; (3) depending on the experimental condition, the target either remained stationary or was displaced during the ocular saccade (due to saccadic suppression, this displacement was not consciously detected by the subjects; Matin 1982); (4) the target was turned off while the next fixation point was turned on (the „target illumination phase“ lasted $1.8 \mathrm{~s}$ ). This sequence was repeated 27 times for each scan (i.e., each scan consisted of a series of 54 targets presented alternatively to the right and to the left). The three experimental conditions were as follows.

\section{Adaptation (ADAPT)}

The initial target appeared $25^{\circ}$ to the right of the fixation point (i.e., the required initial saccadic displacement had a constant amplitude of $25^{\circ}$ ). During the saccadic response, the target location was shifted unbeknownst to the subjects. The shift was consistent within the entire scan. Different intra-saccadic jumps were implemented for each replication of this condition: forward $5^{\circ}$ (scan $\mathrm{ADAPT}_{\mathrm{F} 5} ; 20 \%$ of the amplitude of the first target step); forward $7.5^{\circ}$ (scan $\mathrm{ADAPT}_{\mathrm{F} 7.5} ; 30 \%$ of the amplitude of the first target step); backward $5^{\circ}$ (scan ADAPT $_{\mathrm{B} 5}$ ); backward $7.5^{\circ}$ (scan $\mathrm{ADAPT}_{\mathrm{B} 7.5}$.

\section{Random (RAND)}

This condition was identical to ADAPT, except that the target jump was not consistent from trial-to-trial. The stimulation pattern for each scan was defined by randomly mixing the four types of jumps used in the different replications of the ADAPT condition (forward $5^{\circ}$; forward $7.5^{\circ}$; backward $5^{\circ}$; backward $7.5^{\circ}$ ). When averaged over their different replications, the ADAPT and RAND conditions were balanced from the standpoint of eye kinematics (number, amplitude, and direction of saccades).

\section{Stationary (STAT)}

For this condition, the target remained stationary during the entire trial. It was presented to the right at $17.5^{\circ}\left(\right.$ scan $\left.\mathrm{STAT}_{17.5}\right), 20^{\circ}$ (scan $\operatorname{STAT}_{20}$ ), $30^{\circ}$ (scan $\mathrm{STAT}_{30}$ ), and $32.5^{\circ}$ (scan $\mathrm{STAT}_{32.5}$ ) from the initial fixation point, which corresponded to the final locations reached in the different replications of the ADAPT condition. The target step was constant for a given scan. For each replication, the sequence of target presentation was identical to the one used in the corresponding ADAPT condition (final target). It should be stressed here that STAT and ADAPT were not strictly identical from a kinematic point of view. While the total displacement was strictly balanced in these two conditions, the relative contribution of the primary and corrective secondary saccades was different. In particular, the amplitude and, to a lesser extent, the rate of the corrective saccades was larger in ADAPT than in STAT (see Results).

Each scan was performed in a totally dark room, preventing the subject from using any environmental landmarks. At the end of each scan the light was turned on, and the subject was encouraged ,to stay awake and to look around him“ in order to make sure that any trace of adaptation would disappear before the beginning of the next scan. The inter-scan period was $10 \mathrm{~min}$. The 12 different scans were randomly ordered across subjects.

\section{Behavioral analysis}

Eye movements were recorded binocularly using DC electrooculography at a frequency of $1 \mathrm{kHz}$. During the experiment, eye velocity was extracted on-line from the position signal, using a two-point central-difference derivative algorithm (Bahill and McDonald 1983). The change in target location occurred, in RAND and ADAPT, when eye velocity reached a level equal to half of the peak velocity. The threshold for target jump was set manually on an oscilloscope at the beginning of the experiment while the subject was required to perform a series of $25^{\circ}$ saccades. It was adjusted during the scans if necessary. The technique used for calibrating the EOG signal has been described earlier in detail (Pélisson et al. 1988). In brief, the signal was measured while the subject looked at a sequence of peripheral targets. A calibration curve was then computed by fitting a polynomial through the data. This curve was used to transform the EOG signal into a calibrated eye-position signal. The eye-position signal was numerically filtered at $30 \mathrm{~Hz}$ with a second-order Butterworth dual-pass filter. The beginning and the end of the initial saccade were automatically detected using a velocity-threshold procedure $(50 \%)$. The results of this procedure were checked off-line and corrected, if necessary.

SA generally follows a logarithmic law (Deubel et al. 1986; Straube et al. 1997). In humans, the adaptation takes about 60-80 trials to reach a steady state (Deubel et al. 1986; Frens and Van Opstal 1994). In our experiment, however, the number of trials was limited to 27 per scan, indicating that this steady state was never reached. Since SA presents a roughly linear shape in its initial phase, the time course of adaptation was assessed, for each ADAPT scan, by fitting a normal regression line between the magnitude of the rightward initial saccade and the corresponding trial number (the sum of the squared distance of the observed points to the regression line was minimized). The percentage of adaptation was computed from the slope (s) of the regression line and the magnitude of the intrasacadic jump (IJ) using the following formula $[(\mathrm{s} * 27) / \mathrm{IJ}] * 100$. In order to test the effect of the experimental factors on the adaptation index (slope of the regression lines), an ANOVA with repeated measure was performed $(n=9$ subjects). The repeated-measure factors were the ,jump direction" (backward or forward) and the ,jump amplitude“" $\left(5^{\circ}\right.$ or $\left.7.5^{\circ}\right)$. A mean adaptation index was obtained, for each subject, by averaging the rectified adaptation regression slopes.

Specific analyses were performed for the RAND condition in order to address the possibility that random jumps of the target location generate trial-by-trial adaptive responses. If such were the case, the amplitude of a given saccade should depend on the nature on the previous trial. For instance, if the previous trial were a backward jump, the magnitude of the primary saccade should decrease in comparison to the case where the previous trial was a forward jump. To test this hypothesis, the trials were segregated into two groups. One for which the previous jump was backward and one for which the previous jump was forward. The mean amplitude of the primary saccade was computed for each group and each subject. Paired $t$ statistics were then applied to test whether the primary saccadic response was significantly shorter in the backward than in the forward group.

\section{Imaging}

Imaging methods have been described in previous publications (Winstein et al. 1997; Desmurget et al. 1998). In brief, rCBF images were acquired with a Siemens ECAT Exact scanner by using a modified autoradiographic method in $2 \mathrm{D}$ mode. Scans $(90 \mathrm{~s})$ were recorded every $10 \mathrm{~min}$. The series of scans was made, from each subject, using bolus intravenous injections of $\mathrm{H}_{2}{ }^{15} \mathrm{O}$ (35 mCi), which were delivered $10 \mathrm{~s}$ before the start of the scan. 
Performance of the designated task began at the same time as scanning. Images were reconstructed by using calculated attenuation correction.

Image processing was performed on a SUN Sparc 5 station. For spatial normalization, a within-subject alignment of PET scans was performed by using an automated registration algorithm (Woods et al. 1998a). For each subject, the mean PET image was then co-registered to a population-based PET reference atlas centered in Talairach coordinates (Talairach and Tournoux 1988), using affine and non-linear transforms with 12 degrees of freedom (Woods et al. 1998a, 1998b). Co-registered PET images were smoothed to a final isotropic resolution of $15 \mathrm{~mm}$ and normalized to each other by using proportionate global scaling.

Image analysis

As emphasized in the introduction, the present experiment is bounded by explicit a priori hypotheses. Based on these hypotheses, five search regions were delimited, corresponding to the following structures: the frontal eye field (FEF; bilaterally), the oculomotor cerebellar vermis (OCV), and the superior colliculus (SC; bilaterally). In contrast to the OCV and the SC, which can be easily identified with respect to structural anatomic landmarks, the FEF is not a distinct anatomical region in humans. It is most probably situated around the lateral segment of the precentral sulcus, involving both the posterior extremity of the middle frontal gyrus and the anterior part of the precentral gyrus. In order to define the location of the FEF in a more predictive way, a review of PET studies involving comparison of various oculomotor tasks with a rest or a fixation condition was performed. The search volume was then defined, in Talairach coordinates (Talairach and Tournoux 1988), as the area bounded by the extreme activation loci reported in the literature. As reported in Table 1, this procedure resulted in a quite large area, including the precentral gyrus, the precentral sulcus, and its adjacent inferior and middle frontal gyri. All the areas lying outside the search volume (OCV, SC, FEF) were disregarded in the present study.

Linear-contrast analyses based on the general linear model of ANOVA were used to produce $t$-statistic images between behavioral conditions on a voxel by voxel basis (Neter et al. 1990; Woods et al. 1996). The experimental factors were task, subject, and repetition. In order to privilege sensitivity, we chose a rather lax uncorrected $P$ value $\left(P_{\text {raw }}=0.01\right)$. The $t$-images resulting from the contrast analyses were corrected for multiple comparisons to a final resolution of $P<0.05$, using the method developed by Friston, which takes into account the number of resolution elements constituting the search volume, the size of the activation, and the degree of image smoothness (Friston et al. 1994). Five main contrasts were tested, as follows.

\section{$A D A P T-R A N D$}

This contrast was computed in order to identify the potential contribution of the OCV, the FEF, and the SC to the process of SA. It allowed control for movement kinematics, but may have erased the contribution of some areas showing single-trial corrective effects.

\section{Forward ADAPT-STAT}

For this contrast, the ADAPT scans involving a forward intrasaccadic jump (ADAPT ${ }_{\mathrm{F} 5}, \mathrm{ADAPT}_{\mathrm{F} 7.5}$ ) were compared with the corresponding STAT scans $\left(\mathrm{STAT}_{30}, \mathrm{STAT}_{32.5}\right)$. Although a general contrast involving all the repetitions of the ADAPT and STAT scans was also performed (see below), this forward comparison was carried out considering that both the ADAPT $\mathrm{F}_{\mathrm{F}}$ and STAT trials generate a forward corrective saccade, whereas the $\mathrm{ADAPT}_{\mathrm{B}}$ scans generate a backward corrective saccade. Comparing trials involving backward and forward corrective saccades may have been misleading, considering that the saccadic system is lateralized for the SC (Sparks 1986; Sparks and May 1990) and the FEF (Rivaud et al. 1994; Sommer and Tehovnik 1997).

\section{Kinematic contrast}

As emphasized above, the ADAPT and STAT conditions present kinematic disparities. It is therefore important to determine whether these disparities could explain the pattern of activation observed in the forward ADAPT-STAT contrast. To address this issue, a contrast analysis was performed between the large $\left(32.5^{\circ}\right)$ and small $\left(30^{\circ}\right)$ saccadic displacements $\left[\left(\mathrm{ADAPT}_{\mathrm{F} 7.5}+\mathrm{STAT}_{32.5}\right)\right.$ $\left(\mathrm{ADAPT}_{\mathrm{F} 5}+\mathrm{STAT}_{30}\right)$ ]. It is worth mentioning that the two terms of the contrast (large versus small) vary with respect to the amplitude of the saccadic displacement and the rate of corrective saccades generated (see Results). In view of behavioral data showing that the mean adaptation index is similar in $\mathrm{ADAPT}_{\mathrm{F} 5}$ and $\mathrm{ADAPT}_{\mathrm{F} 7.5}$ (see Results), one may expect the adaptation effect to be erased in the present comparison. If the areas activated in the kinematic contrast were the same as the ones activated in the forward ADAPT-STAT contrast, it would not be possible to determine whether the rCBF increase was related to SA and/or to kinematic factors. However, an absence of activation detected by the kinematic contrast, or an absence of overlap between the areas activated in the kinematic and adaptation contrasts, would support the hypothesis that the sites activated in the forward ADAPT-STAT difference are not related to kinematic disparities.

Table 1 Activation loci observed in earlier positron-emission-tomography studies in the two frontal eye fields (FEF) (adapted and extended from Paus 1996).

\begin{tabular}{|c|c|c|c|c|c|c|}
\hline \multirow[t]{2}{*}{ Study } & \multicolumn{3}{|l|}{ Left FEF } & \multicolumn{3}{|c|}{ Right FEF } \\
\hline & $\mathrm{X}$ & $\mathrm{Y}$ & $\mathrm{Z}$ & $\mathrm{X}$ & $\mathrm{Y}$ & $\mathrm{Z}$ \\
\hline Fox et al. 1985 (reactive vs. rest) & -39 & 7 & 41 & 44 & 11 & 42 \\
\hline Paus et al. 1993 (I) (self-paced vs. fixation) & -23 & 5 & 44 & 24 & 3 & 48 \\
\hline Paus et al. 1993 (II) (reactive vs. rest) & -44 & -6 & 44 & 34 & -2 & 48 \\
\hline Anderson et al. 1994 (reactive vs. fixation) & -24 & -6 & 52 & 20 & -2 & 52 \\
\hline Anderson et al. 1994 (remembered vs. fixation) & -18 & -2 & 52 & 22 & 2 & 48 \\
\hline O'Sullivan et al. 1995 (remembered vs. rest) & -42 & -12 & 48 & 34 & -10 & 44 \\
\hline Paus et al. 1995 (self-paced vs. rest) & -48 & -6 & 50 & 50 & -12 & 47 \\
\hline Petit et al. 1996 (self-paced vs. rest) & -44 & -6 & 40 & 40 & -6 & 40 \\
\hline Petit et al. 1996 (prelearned vs. rest) & -44 & -6 & 40 & 40 & -6 & 40 \\
\hline Sweeney et al. 1996 (reactive vs. fixation) & -22 & -18 & 56 & 38 & -10 & 48 \\
\hline Law et al. 1997 (reactive vs. fixation) & -32 & -12 & 48 & 26 & -10 & 48 \\
\hline Dejardin et al. 1998 (self-paced vs. rest) & -48 & -12 & 40 & 50 & -6 & 32 \\
\hline Extremum & $-48 /-18$ & $-18 / 7$ & $40 / 56$ & $20 / 50$ & $-12 / 11$ & $32 / 56$ \\
\hline
\end{tabular}


ADAPT-STAT, RAND-STAT

In this case, all repetitions were pooled together, for each condition. Because leftward corrective saccades occurred in the ADAPT and RAND conditions, but not in the STAT condition, it is not very informative to test the ADAPT-STAT and RANDSTAT contrasts independently. However, mirroring them could provide worthwhile information. Indeed, the RAND and ADAPT conditions are equivalent from a kinematic point of view. As a consequence, the existence of divergent pattern of activation in the ADAPT-STAT and RAND-STAT contrasts could identify the existence of different functional subsystems in the ADAPT and RAND conditions. Also, a selective activation of a given brain area in one of these two contrasts would strongly suggest that this area was not responding to kinematic disparities.

In the present paper, it was important to rule out the possibility that non-significant activations in the FEF, the SC, or the OCV represent false negative inferences (or type-II errors). To this end, trend analyses were performed for both the ADAPT-RAND and the forward ADAPT-STAT comparisons. The question behind these analyses can be summarized as follows: what is the likelihood that non-significant activations represent false negative inferences? To address this issue, we first determined the voxel for which $\mathrm{rCBF}$ variations had the highest probability of being significant within the area considered (e.g., FEF). To identify this voxel, the $P$ value was raised gradually from the raw threshold $(P=0.01)$ up to a maximum of 0.20 . In a second step, the amplitude of rCBF increase was computed for the identified voxel by substracting the mean blood flow averaged from the RAND or STAT conditions from the mean blood flow averaged from the ADAPT condition (i.e., ADAPT minus RAND, or ADAPT minus STAT at the point of maximal significance). In a third step, the obtained values of rCBF variations (one per subject) were correlated with the behavioral markers. If $\mathrm{rCBF}$ increases were exclusively or mainly related to SA, one would expect a positive correlation based on the premise that the normalized increase in $\mathrm{rCBF}$ should be larger when the degree of adaptation is higher. In a last step, the right and left variations in $\mathrm{rCBF}$ were compared for both the FEF and SC, considering that the contribution of these two structures to saccade generation is lateralized (SC: Sparks 1986; Sparks and May 1990. FEF: Rivaud et al. 1994; Sommer and Tehovnik 1997).

\section{Results}

\section{Behavioral observations: saccade characteristics}

For the STAT condition, the saccadic response consisted of two phases (Fig. 2), as was expected from previous experimental observations (Becker and Fuchs 1969; Prablanc and Jeannerod 1975; Harris 1995): an initial saccade undershooting the target position and a single corrective saccade achieving accurate target acquisition. On average, the initial saccade covered $92 \%$ of the required displacement.

For the RAND condition, the primary saccade represented, on average, $91 \%$ of the initial target jump. This percentage was similar to the one observed in the STAT condition (paired- $t=0.38, P>0.70$ ). The mean amplitude of the first saccade was equal to $23^{\circ}$, indicating that the corrective saccade had a mean angular excursion of either $-3^{\circ}$ (backward $5^{\circ}$ ), $-5.5^{\circ}$ (backward $7.5^{\circ}$ ), $7^{\circ}$ (forward $5^{\circ}$ ) or $9.5^{\circ}$ (forward $7.5^{\circ}$ ). In a majority of trials, we detected only one corrective saccade (Fig. 2). This indicates that the RAND and STAT conditions differed mainly by the respective contributions of the primary
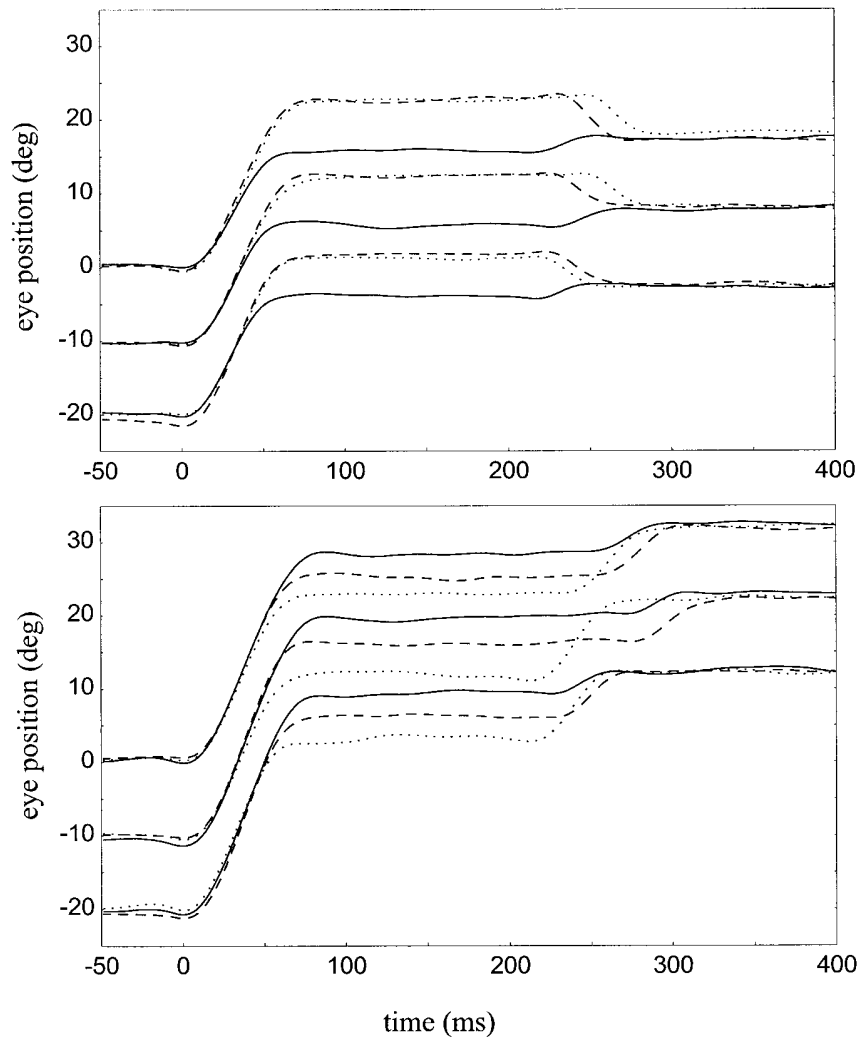

Fig. 2 Eye-position signals for individual saccades performed by one subject in the different experimental conditions (STAT: solid lines; ADAPT: dashed lines; RAND: dotted lines; see Methods section for description of conditions). For the sake of clarity, traces were synchronized with respect to movement onset. They are presented for each of the three starting points. The upper panel displays trials for which the final saccadic displacement had a magnitude of $17.5^{\circ}\left(\mathrm{STAT}_{17.5}, \mathrm{ADAPT}_{\mathrm{B} 7.5}, \mathrm{RAND}_{\text {trials }}\right.$ involving a $7.5^{\circ}$ backward jump). The lower panel displays trials for which the final saccadic displacement had a magnitude of $32.5^{\circ}$ $\left(\mathrm{STAT}_{32.5}, \mathrm{ADAPT}_{\mathrm{F} 7.5}\right.$, RAND trials involving a $7.5^{\circ}$ forward jump). For both panels, the initially required displacement (first target) in RAND and ADAPT had a constant magnitude of $25^{\circ}$. For the upper panel, the trials selected for ADAPT were recorded early in the session. In this case, no difference was observed between the magnitude of the primary saccade for ADAPT and RAND. For the lower panel, trials selected for ADAPT were recorded late in the session. In this case, the magnitude of the primary saccade was slightly larger in ADAPT than in RAND due to saccadic adaptation. As shown in the figure, a single corrective saccade was observed in all conditions. Due to the target jump, however, the correction was larger in RAND and ADAPT than in STAT

and secondary saccades. However, multiple corrective saccades were more frequent in the RAND than in the STAT condition (especially for the large forward target jumps), indicating that these two conditions also differed by the number of saccades generated.

As in the RAND trials, we detected only two saccades in a majority of ADAPT trials (Fig. 2), namely, a main initial saccade and a corrective saccade bringing the gaze on the target. In contrast to RAND, ADAPT generated a progressive change in the amplitude of the primary saccade across the session (Fig. 3). The mean slope of the 

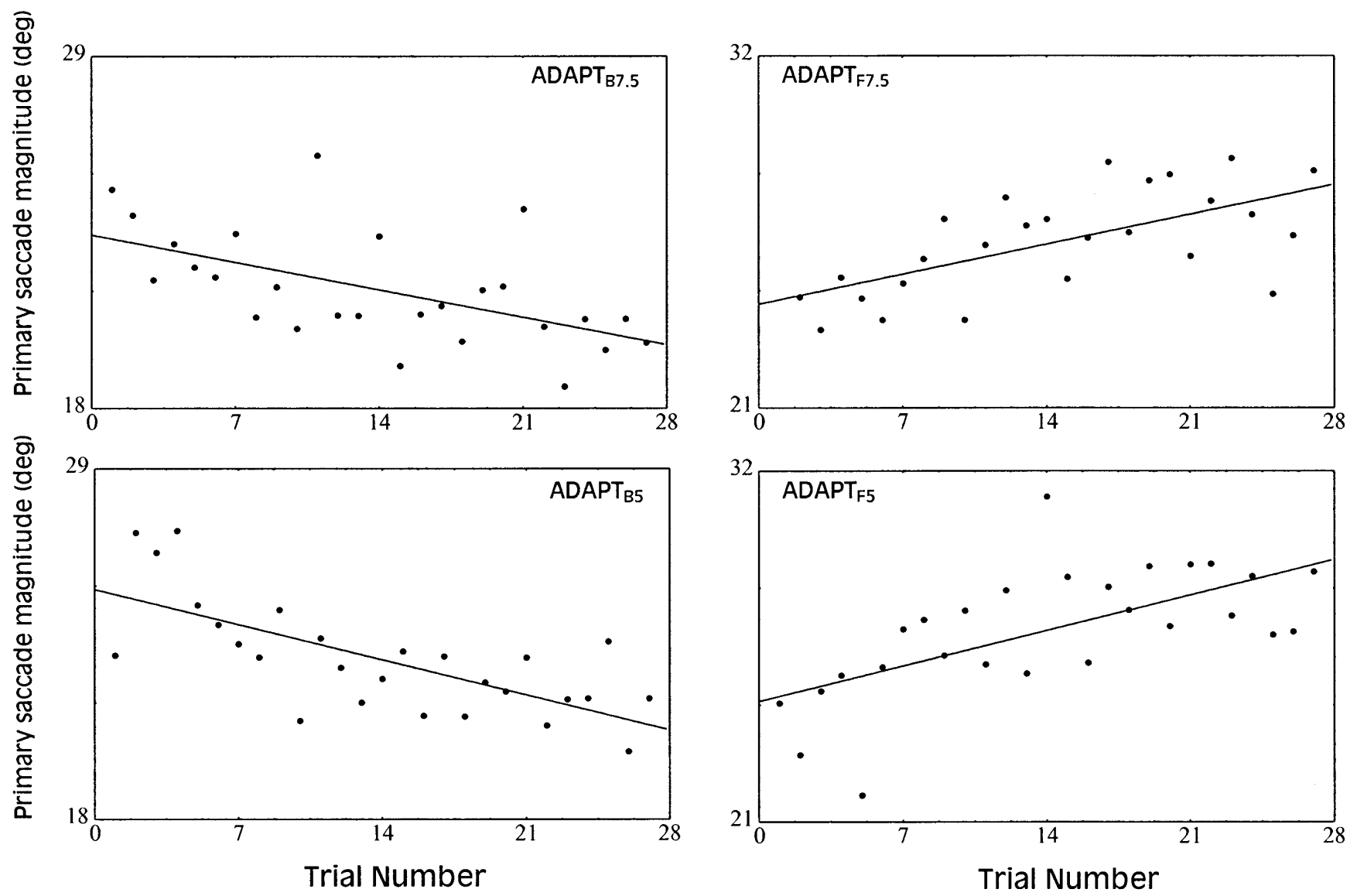

Fig. 3 Illustration of the variations of the saccade magnitude as a function of the trial number for one subject and the four replications of the ADAPT condition (see Methods section for description of condition). Normal regression lines between saccade magnitude and trial number are shown in each panel (solid lines)

normal regression lines was significantly higher than zero for the two forward jump conditions (paired $t$-tests, $P<0.01 ; n=9)$ and significantly lower than zero for the two backward jumps conditions (paired $t$-tests, $P<0.02$; $n=9$ ). As shown by a two-way ANOVA with repeated measures, the adaptation index did not depend on either the amplitude (20\% jumps: 0.09 , 30\% jumps: 0.08; $P>0.75$ ) or the direction (forward jumps: 0.10, backward jumps: $0.07 ; P>0.10$ ) of the intrasaccadic jump. On average, the change in saccadic gain, computed from the regression slopes, represented $39 \%$ of the target jump.

Behavioral observations: trial-by-trial modification of saccade amplitude

In the RAND condition, backward and forward jumps were mixed within the session, generating systematic variations in the post-saccadic error. These variations prevented the oculomotor system from restoring the saccade accuracy over time. Despite this lack of long-term modification of the saccade metrics, a corrective process was present in the RAND condition. The amplitude of

the primary saccade was higher, in eight of the nine subjects, when the previous trial involved a forward jump than when the previous trial involved a backward jump. Statistical analyses showed that the mean difference in saccade magnitude was significant $\left(23.2^{\circ}\right.$ versus $22.6^{\circ}$; $P<0.01)$. It is worth noting here that the RAND condition may lead to an overestimation of the single-trial effect by a factor of 2 . Indeed, when the target jump is random in direction, the saccade lengthening consecutive to a forward jump combines with the saccade shortening consecutive to a backward jump. A more appropriate estimation of the single-trial effect may, thus, be half of the global effect observed in RAND, i.e., $\pm 0.3^{\circ}$. Of interest is the fact that extrapolating this trial-to-trial change of saccadic amplitude to systematic target jumps predicts that the same level of adaptation as in ADAPT should be reached in only about 10 trials $\left(10 \times 0.3^{\circ}=3^{\circ}\right)$. This suggests that the single-trial error-correction mechanism may be a non-additive process, functionally distinct from $\mathrm{SA}$, which acts over a longer time scale. We shall return to this issue in the discussion.

To further address the issue of whether trial-by-trial modification of saccade amplitude and SA could be subserved by common neural processes, the correlation coefficient was computed, across subjects $(n=9)$, between the mean adaptation index (ADAPT) and the value of the trial-by-trial change in saccade amplitude (RAND). The result failed to reveal any significant relation between these two measures $(r=0.29 ; P>0.40)$, contrary to what 
would have been expected if they had a common functional origin. In other words, the subjects who were the „best adaptors" were not the ones who displayed the greatest trial-by-trial effect. It is worth noting that this observation does not rule out the possibility that some areas involved in SA may show trial-to-trial effects or, conversely, that some activations observed in SA are due to the trial-by-trial error correction process.

Because the value of $0.6^{\circ}$ is close to the nominal accuracy of the EOG technique (Pélisson et al. 1988), it is worth mentioning that the existence of a trial-by-trial corrective process has been confirmed in a recent behavioral study using an ISCAN video-recording system (nominal resolution $<0.10^{\circ}$; manuscript in preparation). In this study, seven subjects were requested to perform visually directed saccades having a constant magnitude of $25^{\circ}$. Every eight trials, the target jumped backward during the saccade. In the seven remaining trials, it remained stationary. Results indicated that the saccades performed just after the ,jump“ trials had a significantly shorter amplitude than the saccades performed just before the ,jump“ trials, for all subjects. The mean magnitude of the difference was around $0.2^{\circ}$. In a separate session, SA was induced by jumping the target backward systematically during the saccade. As in the present study, we did not observe any correlation between the

Table 2 Summary of the positron-emission-tomography observations for the different contrasts. Significant correlation coefficients and increases in regional cerebral blood flow $(r C B F)$ that were still significant after adjustments for multiple comparisons are reported in bold characters. Talairach coordinates and increases in rCBF were computed from the voxel of maximal significance. For the structures that did not reveal any significant activation at the highest threshold considered in this study $(P=0.20$; see Methods section), the increase in $\mathrm{rCBF}$ was determined using the Talairach coordinates obtained in the closest related contrast. These structures appear in italics in the table [for the left superior-colliculus rate of adaptation and the amplitude of the trial-by-trial effect $(r=0.25 ; P>0.55)$.

\section{PET observations: ADAPT-RAND}

For the sake of clarity, the main results related to PET observations have been summarized in Table 2. The OCV was the only oculomotor area presenting a significant $\mathrm{rCBF}$ increase when ADAPT was compared with RAND (corrected $t$-images; Fig. 4). The exact cerebellar site of activation was located caudally with respect to the primary fissure (stereotaxic coordinates for the voxel of highest significance: $4,-69,-19)$. It encompassed the cerebellar lobules VI and VII bilaterally. The metabolic increase was more marked on the right side, i.e., on the side ipsilateral to the direction of the adapted saccadic displacement. The mean $\mathrm{rCBF}$ increase observed in ADAPT with respect to RAND was equal to $2.3 \%$. The vermal activation was very robust and not related to the rather lax, uncorrected $P$ value adopted in the present experiment. A significant metabolic increase was still observed on the corrected $t$-images when $P_{\text {raw }}$ was lowered to 0.0001 . Further evidence suggesting that this increase was really related to SA was provided by the demonstration that the subjects who were the „best adaptors“ were

(SC) ADAPT-RAND contrast, coordinates were inferred from the forward ADAPT-STAT contrast; for the right SC ADAPT-RAND and forward ADAPT-STAT contrasts, coordinates were determined by symmetry from the left SC forward ADAPT-STAT contrast; for the right SC ADAPT-STAT contrast, coordinates were determined by symmetry from the left SC ADAPT-STAT contrast; for the left frontal eye fields $(F E F)$ ADAPT-STAT contrast, coordinates were determined by symmetry from the right FEF ADAPT-STAT contrast; see Methods section for description of conditions]. $O C V$ Oculomotor cerebellar vermis

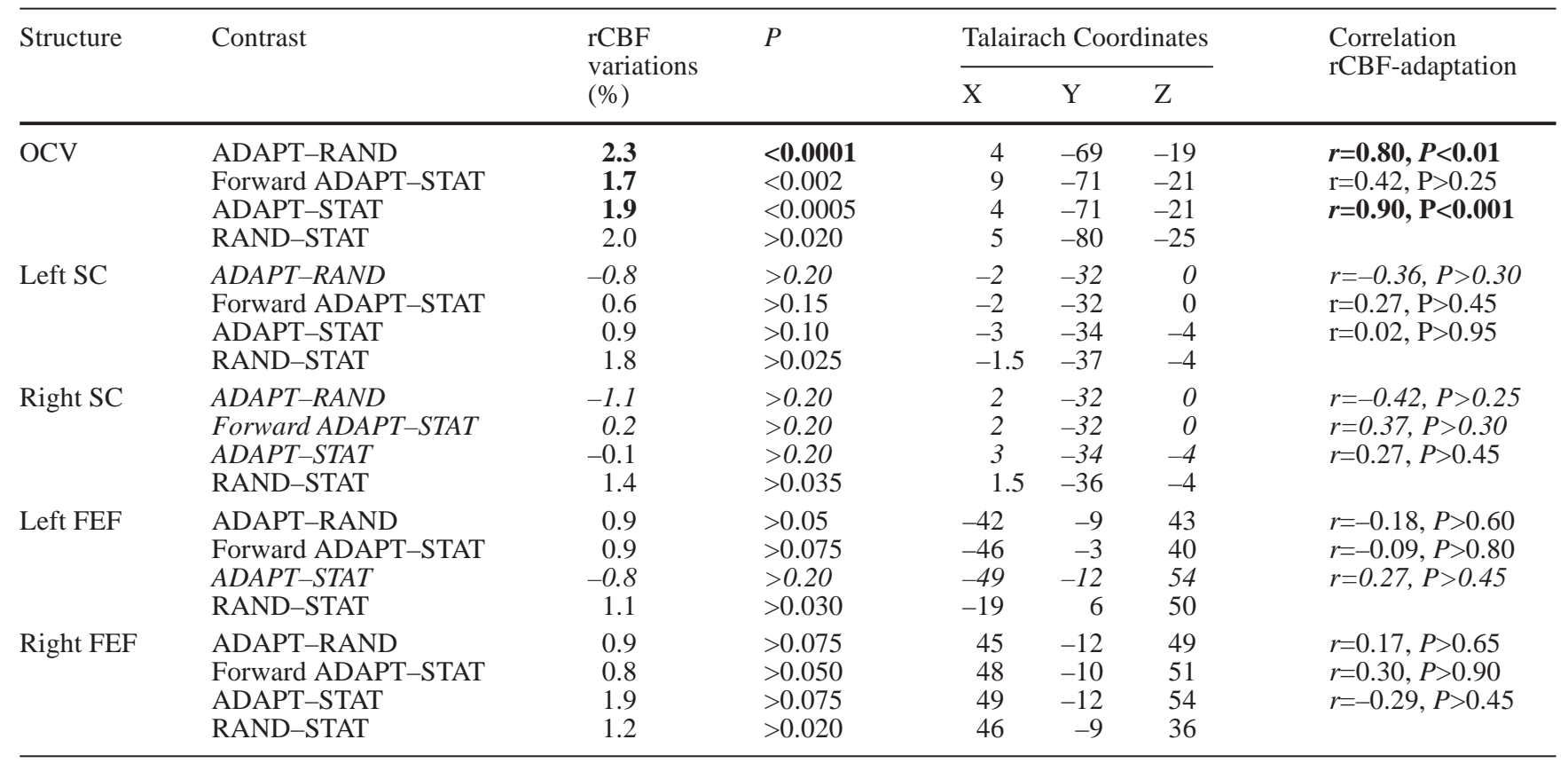




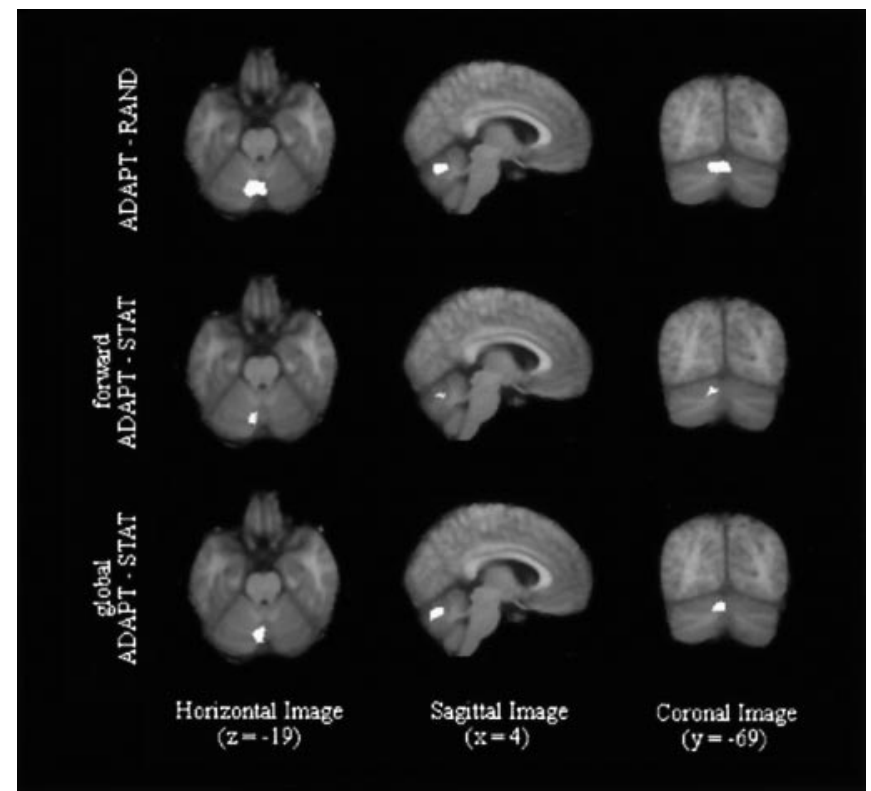

Fig. 4 Implication of oculomotor cerebellar vermis (OCV) in the process of saccadic adaptation. The figure displays the horizontal (left column), sagittal (middle column), and coronal (right column) mean difference images obtained after correction for multiple comparisons in the ADAPT-RAND, forward ADAPT-STAT, and global ADAPT-STAT contrasts ( $n=9$ subjects; see Methods section for description of conditions). Images are centered on the point of maximal significance observed in the ADAPT-RAND contrast. The right side is shown on the left of horizontal and coronal images

also the ones who displayed the highest rCBF increase. Indeed, a significant correlation was observed between the mean adaptation index and the $\mathrm{rCBF}$ increase in the OCV ( $r=0.80 ; P<0.01$; Fig. 5$)$.

No significant activation was observed within the FEF areas at the canonical threshold (corrected or uncorrected $t$ images). To address the possibility that this result was a false-negative inference, the voxels presenting the most significant changes in $\mathrm{rCBF}$ were determined in both FEF by progressively increasing the $P$ value (left FEF: $-42,-9$, 43; right FEF: 45, -12, 49). For the identified regions, the metabolic activation presented four particularities. First, the statistical probability was higher than $P=0.05$. Second, the $\mathrm{rCBF}$ increase was lower than $0.9 \%$ (left $0.89 \%$, right $0.87 \%$ ). Third, no correlation was observed between the rCBF variations in the FEF and the mean adaptation index (left: $r=-0.18, P>0.60$; right: $r=0.17, P>0.65$ ). This point is illustrated in Fig. 5, which shows that a strong adaptive response can be associated with either a decrease (e.g., Fig. 5, subject $\square$ presenting the second highest adaptive effect) or an increase (e.g., Fig. 5, subject $\bigcirc$ presenting the highest adaptive effect) in rCBF in the FEF areas. Fourth, metabolic changes in the right and left FEF were found to be statistically equivalent (paired $t=0.06 ; P>0.95$ ). This is not consistent with the lateralized contribution of the FEF in the control of saccadic eye movements. Indeed, it has been shown that the FEF controls the amplitude of sac-
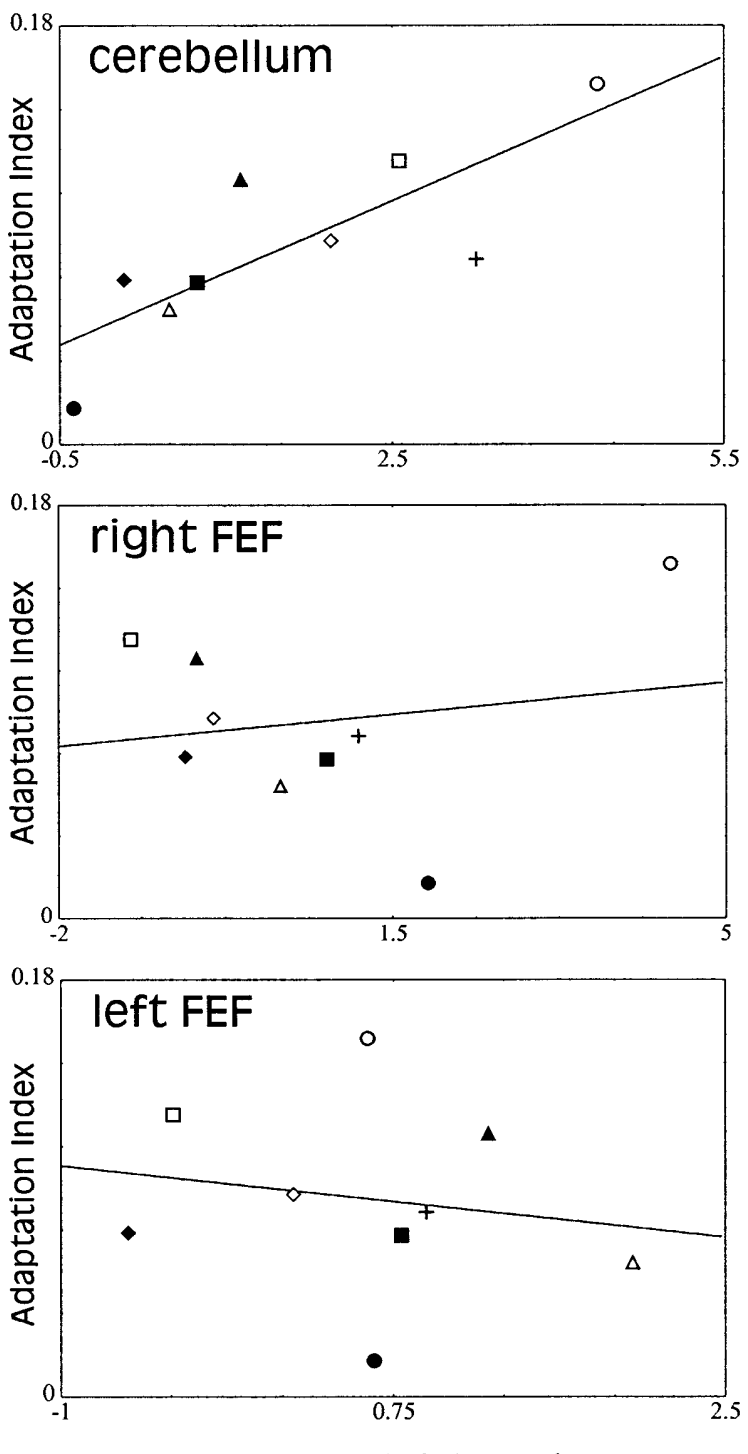

rCBF change (ml/min/100g tissue)

Fig. 5 Illustration of the inter-individual relationship linking the mean adaptation index and the corresponding regional cerebralblood-flow $(r C B F)$ change attributable to the adaptation effect (,ADAPT“ minus „RAND“). A correlation between these two parameters was found for the cerebellum $(r=0.80 ; P<0.01)$, indicating that the subjects who were the best ,adaptors“ also displayed the largest increases in $\mathrm{rCBF}$. No correlation was observed for either frontal eye field $(F E F)$ (left FEF: $r=-0.18$; right FEF: $r=0.17$; $P>0.60)$. The continuous lines shown in the figure represents the normal regression lines. For the sake of clarity, each subject is represented with specific symbols on the figure

cadic displacements performed in the contralateral direction (Bruce and Goldberg 1985; Bruce et al. 1985; Schall 1991; Rivaud et al. 1994; Sommer and Tehovnik 1997). Because only the amplitude of rightward saccades was modified in the present study, one may have predicted that potential rCBF changes related to SA should have occurred in the left FEF, but not in the right FEF. When considered together, all four previous observations do not substantiate the idea that the metabolic variations observed in the FEF were relevant in terms of SA. 
Finally, we did not detect any significant activation in the right or left SC, even when the $P$ value was raised to 0.20 . With respect to this point, it may be worth mentioning that a slight decrease in $\mathrm{rCBF}$ was in fact observed in both the left and right SC when ADAPT was compared with RAND (Table 2).

\section{PET observations: forward ADAPT-STAT}

The OCV was the only area which presented a significant $\mathrm{rCBF}$ increase when ADAPT was compared with STAT $(9,-71,-21$; corrected $t$-images, Fig. 4). This result was not related to the relatively high raw $P$ value adopted in the present experiment. Indeed, a significant activation increase was still observed, for the OCV, on the corrected $t$-images when $P_{\text {raw }}$ was lowered to 0.002 . The mean rCBF increase observed in ADAPT with respect to STAT was equal to $1.7 \%$.

Two arguments suggest that the cerebellar activation observed in the forward ADAPT-STAT contrast was not associated with kinematic factors, but instead with the process of SA. First, the site of activation overlapped consistently with the one observed in the ADAPTRAND contrast, where kinematic differences were controlled. Second, cerebellar activity was not responsive to small variations in saccade characteristics. Indeed, neither the kinematic contrast nor the RAND-STAT contrast (see below) showed a significant $\mathrm{rCBF}$ increase in the vermal area. Note that, although positive, the correlation coefficient between the rCBF increase and the degree of adaptation did not reach the statistical threshold $(r=0.42, P>0.25)$.

As in ADAPT-RAND, no significant activation was observed within the FEF areas at the canonical threshold (corrected or uncorrected $t$ images). To address the possibility that this result was a false-negative inference, the voxels presenting the most significant changes in $\mathrm{rCBF}$ were determined in both FEF by progressively increasing the $P$ value (left FEF: $-46,-3$, 40; right FEF: 48, $-10,51)$. For both FEF, the rCBF increase was less than $0.9 \%$ (left $0.89 \%$, right $0.75 \%$ ). The probability of the increase being significant was higher than $P=0.05$ in both FEF. In addition, the changes in $\mathrm{rCBF}$ were clearly not correlated with behavioral observations. No relation was observed between the rCBF variations in the FEF and the mean adaptation index (left: $r=-0.09, P>0.80$; right: $r=0.3, P>0.90)$. As shown by further analyses, the rCBF increase was not significantly different in the two FEF (paired $t=0.32, P>0.75$ ), contrary to what would have been expected if metabolic changes were really related to SA (see previous section). These observations do not support the interpretation that the metabolic variations observed in the FEF were relevant in terms of SA.

A slight increase in rCBF was observed in the left SC $(-2,-32,0)$ when ADAPT was compared with STAT $(0.64 \%)$. The probability of this increase being significant was quite low $(P>0.15)$. No correlation was observed between $\mathrm{rCBF}$ variations in the left $\mathrm{SC}$ and the mean adaptation index $(r=0.27 ; P>0.45)$. The $\mathrm{rCBF}$ variations observed in the right $\mathrm{SC}$ did not reach the significance level, even for the highest statistical threshold considered in this study $(P=0.20)$. This result is not surprising, considering that the $\mathrm{rCBF}$ increase observed in the right SC was quite small $(0.15 \%$ at location $2,-32,0)$. As for the FEF, variations in $\mathrm{rCBF}$ observed within the left and right SC were not statistically different (paired $t=1.2, P>0.25$ ), as would have been expected if some metabolic changes in the left SC were related to SA. Indeed, as with the FEF, the SC is involved in the generation of saccadic displacements performed in the contralateral direction (Sparks 1986; Sparks and May 1990).

\section{PET observations: ADAPT-STAT, RAND-STAT}

The ADAPT-STAT contrast revealed a large activation in the OCV (Fig. 4). The activation site $(4,-71,-21)$ was close to the one observed in the ADAPT-RAND and the forward ADAPT-STAT contrasts. The mean rCBF increase observed in ADAPT with respect to STAT was equal to $1.9 \%$. As in the ADAPT-RAND condition, a significant correlation was observed between the mean adaptation index and the rCBF increase in the OCV $(r=0.90 ; P<0.001)$. No significant vermal activation was observed in the RAND-STAT comparison (mean rCBF decreased by a marginal amount in RAND with respect to STAT at location $4,-71,-21 ; 0.04 \%$ ), suggesting that the significant activation observed in the ADAPT-STAT contrast was not related to kinematic factors.

No activation was observed in the FEF and the SC for the ADAPT-STAT contrast (corrected or uncorrected $t$ images). This result is congruent with our previous observations showing an absence of activation within these areas for the ADAPT-RAND and forward ADAPTSTAT contrasts.

As in the ADAPT-STAT contrast, the RAND-STAT contrast failed to reveal any significant activation within the FEF or the SC (corrected or uncorrected $t$-images). We shall return on this point in the discussion.

\section{Discussion}

The main aim of the present paper was to address the functional anatomy of SA in humans. Based on a review of the literature, we focused on the three principal structures that have been suggested as potential loci for SA, namely the FEF, the SC, and the OCV. Our results indicate a clear involvement of the latter area. We found no evidence to suggest that the FEF and the SC are also recruited during SA. Besides these main findings, we also showed that saccade metrics were controlled on a trialby-trial basis in the RAND condition. We propose designating this process single-trial error correction (STEC). Our data suggest that STEC does not rely on the cerebellum and is functionally different from the process of SA. From the present study, however, it remains unclear how 
STEC and SA interact with each other. Also, it is not established whether the observed trial-by-trial modulation of saccade amplitude represents a general process or a specific response to the (unusual) RAND condition.

\section{Oculomotor cerebellum mediates SA in humans}

Numerous experiments involving single-unit recordings (Kase et al. 1980; Ohtsuka and Noda 1995), brain lesions (Optican and Robinson 1980; Robinson et al. 1993; Goffart and Pélisson 1998; Takagi et al. 1998; Barash et al. 1999), electrical stimulation (Ron and Robinson 1973; Keller et al. 1983; Fujikado and Noda 1987; Noda and Fujikado 1987), or imaging (Petit et al. 1993; Sweeney et al. 1996; Dejardin et al. 1998) have indicated that the OCV is critically involved in the control of saccadic eye movements. Human studies implicating magnetic stimulation of the vermis in normal subjects (Hashimoto and Ohtsuka 1995) or patients with various degrees of cerebellar disorders have suggested that this region may be particularly important in the control of saccade metrics. With respect to the latter point, it was shown that large lesions of the medioposterior cerebellum did not prevent subjects from performing saccades, but did result in marked saccadic dysmetria (Zee et al. 1976; Lewis and Zee 1993).

Systematic microstimulation studies in monkeys have demonstrated that the portion of the cerebellar cortex involved in saccade control is located in lobules VI and VII (Fujikado and Noda 1987; Noda and Fujikado 1987). When these lobules are lesioned (Sato and Noda 1992; Takagi et al. 1998; Barash et al. 1999) or microstimulated during saccadic displacement (Ohtsuka and Noda 1991a), monkeys consistently make dysmetric saccades. Apparently, the oculomotor vermis can exert a rather direct control on the brainstem saccadic generator. Microstimulation delivered in the OCV elicits ipsilateral saccades with latencies of $15 \mathrm{~ms}$ (Fujikado and Noda 1987). This influence seems to be routed through the fastigial complex. As shown by Noda and colleagues, the Purkinje cells of the OCV send an inhibitory projection to a restricted area in the caudal part of the fastigial nucleus (Yamada and Noda 1987; Ohtsuka and Noda 1991a, 1991b). This fastigial oculomotor region (FOR), which in turn projects to the thalamus (Asanuma et al. 1983), to the SC (May et al. 1990), and to specific oculomotor regions in the brainstem (Noda et al. 1990), seems to carry information for controlling the amplitude of the saccade. Because bilateral inactivation or lesioning of the FOR results in extreme saccadic hypermetria (Optican and Robinson 1980; Robinson et al. 1993; Straube et al. 1995), it is generally thought that this region modulates an oversized oculomotor command issued by the brainstem saccadic generator by influencing premotor neurons of the reticular formation and/or saccadic neurons of the SC (Optican and Robinson 1980; Robinson et al. 1993; Ohtsuka and Noda 1991b; Dean et al. 1994; Schweighofer et al. 1996; Goffart and Pélisson 1998; Lefèvre et al. 1998; Guillaume and Pélisson 1999). According to this view, FOR sends a corrective signal that is added to the brainstem command in order to ensure a proper match between the characteristics of the motor output and the amplitude of the required displacement.

In summary, the previous observations indicate that the nervous system can modulate the amplitude of the ocular saccade by tuning the inhibitory action exerted by the vermal Purkinje cells on the fastigial nuclei. Our observations suggest that one of the functions subserved by this modulation, in humans, is to maintain proper calibration between the sensory and motor components of the saccadic system (in other words, to achieve SA). In support of this hypothesis, we showed that PET difference images, reflecting metabolic changes attributable to the process of SA, involved a large activation within the OCV. To avoid ambiguity, it may be worth emphasizing at this point that the rCBF effects of backward and forward jumps do not cancel each other. If such had been the case, i.e., if backward adaptation induced a decrease in $\mathrm{rCBF}$ within the OCV while forward adaptation induced an increase, no significant activation should have been observed for the ADAPT-RAND and global ADAPT-STAT contrasts (the ADAPT condition involved adaptation to backward and forward jumps). The vermal activation observed for the ADAPT-RAND and global ADAPT-STAT contrasts shows that the metabolic activation in the OCV is reflective, not of the level of inhibition itself, but of the adaptive process modulating the inhibition level exerted by the Purkinje cells. The present results are consistent with previous reports showing an impairment of SA capacities in human patients suffering from syndromes associated with cerebellar dysfunctions (Waespe and Baumgartner 1992). Also, they are in agreement with studies in monkeys showing that lesions of the OCV (Takagi et al. 1998; Barash et al. 1999) and/or the cerebellar fastigial nuclei (Optican and Robinson 1980; Goldberg et al. 1993) prevented SA from occurring.

Metabolic activity does not change significantly in the FEF during SA

PET difference images, reflecting $\mathrm{rCBF}$ changes attributable to the process of SA, did not reveal significant metabolic changes within the FEF. Further analyses were performed to determine whether this conclusion represented a false-negative inference. These analyses indicated that $\mathrm{rCBF}$ variations within either FEF were not related to the process of behavioral adaptation. They also showed that the magnitude of $\mathrm{rCBF}$ increases remained small in both FEF. This latter finding contrasts with the observation that the FEF are readily recruited during oculomotor tasks (previous PET studies involving various oculomotor tasks have invariably shown a fairly robust saccade-related activation within the FEF areas; Table 1; Pierrot-Desiligny et al. 1995; Paus 1996). Finally, our data indicated that $\mathrm{rCBF}$ changes did not differ in the 
right and left FEF, contrary to what would have been predicted if these areas were crucially involved in the process of SA. Indeed, the FEF has been shown to control contralaterally directed saccades (Bruce and Goldberg 1985; Bruce et al. 1985; Schall 1991; Rivaud et al. 1994; Sommer and Tehovnik 1997). As a consequence, because only the amplitudes of rightward saccades were modified in the present study, one would have predicted that any SA-related changes in $\mathrm{rCBF}$, for the FEF, would have engaged the left hemisphere exclusively and not the right one.

Neither the RAND-STAT contrast nor the ADAPTSTAT contrast revealed a significant $\mathrm{rCBF}$ increase within the FEF. This is puzzling, especially for the right FEF, because the number of leftward corrective saccades was much larger in the RAND and ADAPT conditions (50\%) than it was in the STAT condition (0\%). The absence of activation within the right FEF might be related to the inability of the PET technique to detect blood-flow variations associated with small saccadic responses (as reported in the Results section, leftward corrective saccades had an average amplitude of around $5^{\circ}$ in the present study). Even if we cannot unequivocally reject this possibility, we think that it is quite unlikely. Indeed, as already mentioned, saccade-related activations are known to be fairly robust in the FEF, even for small displacements. For instance, Paus et al. (1993) detected significant activations within both FEF during oculomotor tasks involving saccadic displacements of $5^{\circ}$. An alternative explanation for the absence of FEF activation in the ADAPT-STAT and RAND-STAT contrasts may be that the execution of corrective saccades does not significantly engage the FEF. This hypothesis is in accord with behavioral studies showing that corrective saccades occur at consistently shorter latencies than the primary saccades (Becker and Fuchs 1969; Prablanc and Jeannerod 1975), suggesting that they may rely more on subcortical structures. Also, it relates to a recent observation in a patient exhibiting a localized ischemic lesion in the left FEF (Rivaud et al. 1994). When this patient was required to perform memory-guided saccades, reflexive saccades (gap paradigm), or predictive saccades to the right, a significant hypometria was systematically observed. Interestingly, in a double-step paradigm, this hypometria was present for the primary saccade, but not for the secondary saccade. Rivaud et al. argued that the amplitude of the memory-guided saccades, the reflexive saccades, the predictive saccades, and the primary saccades in the double-step paradigm was defined in retinotopic coordinates. By contrast, they claimed that the computation of the amplitude of the secondary saccade in the double-step task was based on both retinal and extra-retinal signals. Following these premises, one may hypothesize that the FEF is mainly involved in the generation of primary retinotopic saccades, but not in the generation of secondary corrective saccades (see also, for a discussion, Pierrot-Deseilligny et al. 1995). This could explain why no FEF activation was observed in the present study, where kinematic differences depended mostly on secondary corrective saccades.
Recent psychophysical experiments have shown, in humans, that SA does not transfer from reactive to selfgenerated saccades (Erkelens and Hulleman 1993; Deubel 1995). As underlined by Deubel (1995, p 3538) „it may be tempting to speculate that the neural substrate of the reflexive system might be the rather direct retinocollicular pathways (...), while the substrate for the intentionally controlled saccades may include the frontal eye fields". In agreement with this view, Straube et al. (1995) provided evidence that cerebellar lesions may affect reactive saccades, but not self-generated saccades. As shown by these authors, a patient presenting with bilateral ablation of the fastigial nuclei exhibited large deficits when required to perform reactive saccades to jumping targets. In conformity with monkey studies (Optican and Robinson 1980; Goldberg et al. 1993), it was observed that the patient dramatically overshot the target 6 days after surgery. Interestingly, no sign of hypermetria was observed for self-generated saccades performed toward stationary targets, suggesting that the cerebellum was not critically involved in the generation of these saccades. In parallel with this result, it has been suggested that the FEF may be more involved in the control of self-generated saccades than in the control of reflexive saccades. With respect to this hypothesis, it was shown, in monkeys, that lesions of the FEF did not dramatically impair the accuracy of contralateral reactive saccades performed to jumping targets that remained illuminated until foveation was achieved (Schiller et al. 1980; Lynch 1992; Sommer and Tehovnik 1997). In humans, it was observed that localized FEF lesions did not consistently increase saccadic latency in a gap task (Pierrot-Deseilligny et al. 1991; Rivaud et al. 1994), contrasting with the large impairment noted for intentional saccades, such as anti-saccades, memory-guided saccades, or predictive saccades. Also, in the FEF, the rCBF increase was found to be significantly greater in memory-guided saccades than in reactive saccades (Sweeney et al. 1996). When considered together, the previous results leave open the possibility that the FEF and the cerebellum may be more critically involved in the generation of volitional and reactive saccades, respectively. As a consequence, one may not exclude the hypothesis that the selective cerebellar activation observed in the present study was task specific. It is possible that adaptation of self-generated saccades would have involved additional or different structures, including the FEF.

\section{Metabolic activity does not change significantly} in the SC during SA

In the present study, we did not find evidence that the SC was activated during SA. Because earlier imaging studies have shown that PET cameras were not always successful at capturing kinematic-related $\mathrm{rCBF}$ increases within the SC (for a counter-example, see Paus et al. 1995), this result should be interpreted cautiously. However, beyond this reservation, one should mention that 
our negative observation fits well with electophysiological studies showing, in monkeys, that collicular motor cells do not modify their pattern of discharge after SA, even when the adapted saccade no longer terminates in the original movement field (Goldberg et al. 1993; Frens and Van Opstal 1997; see Introduction).

Single-trial modification of the saccade amplitude

In the RAND condition, we observed that the metrics of the current saccade varied as a function of the error observed in the previous saccade. When the previous saccade overshot the target, the next saccade tended to be of shorter amplitude. By contrast, when the previous saccade undershot the target, the next saccade tended to be of larger amplitude. Earlier in the discussion, we proposed naming this corrective process single-trial error correction (STEC).

The first question that needs to be addressed in this section concerns the relation between SA and STEC. Two hypotheses are plausible. On the one hand, on may speculate that STEC represents a specific functional process independent of SA. On the other hand, on may assume that STEC is constitutive of SA. According to this latter view, adaptation would represent the arithmetic sum of all the successive trial-by-trial modifications of the saccadic gain. Three arguments, based on our data, favor the independence hypothesis. First, the magnitude of the trial-by-trial effect was much larger than what would be expected if STEC represented the functional basis of SA. Second, no correlation existed between the mean adaptation index and the amplitude of the trial-bytrial effect. In other words, the subjects who presented the largest trial-by-trial response were not the ones who adapted the fastest, indicating that the magnitude of STEC does not predict the celerity of SA. Third, PET images were not consistent with the view that SA results from the addition of incremental corrections occurring on a trial-by-trial basis (STEC). Indeed, if such was the case, the net activation observed, with respect to the STAT condition, should be the same in the OCV for the ADAPT and RAND conditions, predicting that: (1) a vermal activation should be observed in the RANDSTAT contrast (STEC), and (2) no vermal activation should be observed in the ADAPT-RAND contrast. The actual data show that the oculomotor vermis was activated only in association with SA, a result opposite to the above predictions.

Although SA and STEC may represent independent functional processes, they are both designed to preserve the accuracy of the oculomotor system. With respect to this point, it has been shown in numerous studies, including the present one, that reactive saccades to targets presented in the peripheral visual field consist of two phases: an initial response undershooting the target position and a single correction achieving accurate target acquisition (Becker and Fuchs 1969; Prablanc and Jeannerod 1975; Harris 1995). Strikingly, when the target is displaced backward during the initial oculomotor response in such a way that the postsaccadic error is null at the end of the primary saccade, the system adapts to restore the initial undershoot (Henson 1978), indicating that hypometria is actively maintained in the face of experimental perturbations. Although the reason for a systematic and deliberate undershoot is not understood yet (for a discussion of this point, see Robinson 1973; Optican 1982; Becker 1989; Harris 1995), the previous observations suggest that neither STEC nor SA are aimed at nullifying the post-saccadic error. We rather believe that both STEC and SA are aimed at maintaining the saccadic gain within an optimal range. This idea fits, for instance, with the assumption that setting the saccadic gain just below unity allows minimization of the total saccadic flight-time. As shown by Harris (1995), the optimal gain that would minimize the time spent in saccade would be around 0.93 (i.e., the initial saccade covers $93 \%$ of the required displacement), a value that is very close to the one observed in the present study (0.92).

If SA and STEC both serve to keep the saccadic gain around its optimal range, and if, as previously postulated, these two processes represent independent functional entities, one may wonder about their raison d'être. Indeed, why would the nervous system use two different adaptive processes to do the same thing? Although this issue cannot be addressed unequivocally from our data, a plausible explanation may be that SA and STEC process different types of errors and work on different time scales. As many functional systems, the saccadic system has to cope with two different types of errors:

1. The systematic errors which result from permanent changes within the oculomotor system. These errors are generally of large magnitude, and they can be related, for instance, to neural death, brain lesions, or developmental modifications in the biomechanical characteristics of the oculomotor apparatus.

2. The variable errors which result from transient changes within the oculomotor system. These errors are generally of limited magnitude and they can result, for instance, from stress, fatigue, or variations in the transmission of the neural signal.

From the data published in the literature, one can predict that SA is functionally incapable of correcting for variable errors. Indeed, even if SA may take place quickly in certain experimental contexts (Deubel et al. 1986; Frens and Van Opstal 1994), it develops very slowly under normal conditions where the number of possible saccadic displacements is not restrained to a small array of targets (Scudder et al. 1998). Restoration of saccadic accuracy requires, for instance, several days in patients presenting a monocular paresis of the extraocular muscles (Kommerell et al. 1976; Optican et al. 1985). The use of a long-term adaptive process to deal with transient errors would be, at best, inefficient and, at worse, hazardous for the stability of the oculomotor system. Because of its instantaneous reactivity, STEC does not present these drawbacks, and it may represent an appropriate correc- 
tive process to deal with transient errors. This assumption seems all the more plausible considering that variable errors are expected to be small and potentially of an order of magnitude compatible with the $\pm 0.3^{\circ}$ variations observed for STEC in the present study. Because it works on a single-trial basis, we propose that STEC does not involve any sensorimotor remapping within the neural oculomotor circuitry, as SA does. We suggest, as a heuristic hypothesis, that STEC operates like a feedforward control system, in which the postsaccadic error observed in the previous saccade is used to modulate the gain of the forthcoming saccadic command. According to this view, STEC would substitute control signals that produce the correct response for control signals producing an incorrect response (Jordan and Rosenbaum 1989; Jordan 1990). In support of this model, it may be worth reporting that a functional dissociation between a feedforward, trial-by-trial corrective mechanism and a longterm adaptive process has been recently identified for pointing movements in several studies dealing with prismatic adaptation (Rossetti et al. 1993; Rossetti 1994; Redding and Wallace 1996).

From our data, it was not possible to identify the functional substrate of STEC. The absence of activation observed in the RAND-STAT contrast can be accounted for by two different factors. On the one hand, it may be that STEC relies on oculomotor structures other than the ones investigated in the present study. On the other hand, it is possible that variations in $\mathrm{rCBF}$ associated with STEC are too modest to be detected by PET cameras. Three elements may contribute to this. First, STEC may rely on small subcortical structures, like the SC or the FOR, which are not easily detectable by PET cameras. Second, the metabolic signal related to STEC may be small and hard to detect, considering that this process is likely to contribute to the maintenance of saccadic accuracy also in the STAT condition. Third, the RAND and STAT conditions exhibit kinematic differences that may hide the potential effect of STEC. To illustrate this point, let us assume that the left SC is involved in controlling saccade accuracy on a trial-by-trial basis (jump only occurred for rightward primary saccades in the present study). In this case, the rCBF would be expected to increase in the left SC for the RAND-STAT contrast. However, the number of rightward corrective saccades was larger in STAT (100\%) than in RAND (50\%), which may have resulted in a higher activation within the left SC for the STAT condition. This kinematic effect may partially mask the STEC effect. These issues may be better controlled for in the future using event-related fMRI for instance (Rosen et al. 1998).

\section{Conclusions}

The main aim of this study was to identify the functional substrate of SA in human. We obtained two main results. First, we showed, in a target-jump paradigm, that the progressive modification of the saccade amplitude was mediated by the OCV. Second, we provided evidence that the contributions of the FEF and the SC to this adaptive process were marginal, if any. These findings are congruent with previous neurophysiological studies in monkeys, suggesting that SA shares similar neural circuits in human and animal. Beyond this conclusion, however, several issues remain to be addressed. For instance, it is not clear what the functional anatomy of SA would be for non-reactive saccades, knowing that saccadic gain modifications do not transfer from one type of saccade (e.g., reactive) to another (e.g., volitional). Also, it is not established whether the cerebellar activation we observed in the present study was reflective of the adaptive state itself (modification of the output signal sent by the OCV to the fastigial nucleus) or only of the adaptive process (e.g., a „teaching signal“ driving modifications of the fastigial synaptic connectivity). Further experiments are needed to address these questions.

Acknowledgements We thank Michael White and Delicia Votaw for their technical assistance and Roger Woods and John Votaw for providing image-analysis software. We also thank Mike Crutcher, Laura Payne, and Garry Russo for their helpful remarks and their critical reading of the manuscript. This project was supported by the US Public Health Service.

\section{References}

Albano JE (1996) Adaptive changes in saccade amplitude: oculocentric or orbitocentric mapping? Vision Res 36:2087-2098

Anderson TJ, Jenkins IH, Brooks DJ, Hawken MB, Frackowiak RS, Kennard C (1994) Cortical control of saccades and fixation in man. A PET study. Brain 117:1073-1084

Asanuma C, Thach WR, Jones EG (1983) Anatomical evidence for segregated focal groupings of efferent cells and their terminal ramifications in the cerebellothalamic pathway of the monkey. Brain Res 286:267-297

Bahill AT, McDonald JD (1983) Frequency limitations and optimal step size for the two-points central difference derivative algorithm with application to human eye data. IEEE Trans Biomed Eng 30:191-194

Barash S, Melikyan A, Sivakov A, Zhang M, Glickstein M, Thier P (1999) Saccadic dysmetria and adaptation after lesions of the cerebellar cortex. J Neurosci 19:10931-10939

Becker W (1989) Metrics. In: Wurtz RH, Goldberg ME (eds) The neurobiology of saccadic eye movements. Elsevier, Amsterdam, pp 13-67

Becker W, Fuchs AF (1969) Further properties of the human saccadic system: eye movements and correction saccades with and without visual fixation points. Vision Res 19:1247-1258

Bruce CJ, Goldberg ME (1985) Primate frontal eye fields. I. Single neurons discharging before saccades. J Neurophysiol 53:603-635

Bruce CJ, Goldberg ME, Bushnell MC, Stanton GB (1985) Primate frontal eye fields. II. Physiological and anatomical correlates of electrically evoked eye movements. J Neurophysiol 54:714-734

Dean P, Mayhew EW, Langdon P (1994) Learning and maintaining saccadic accuracy: a model of brainstem-cerebellar interactions. J Cogn Neurosci 6:117-138

Dejardin S, Dubois S, Bodart JM, Schiltz C, Delinte A, Michel C, Roucoux A, Crommelinck M (1998) PET study of human voluntary saccadic eye movements in darkness: effect of task repetition on the activation pattern. Eur J Neurosci 10:2328-2336

Desmurget M, Pélisson D, Urquizar C, Prablanc C, Alexander G, Grafton ST (1998) Functional anatomy of saccadic adaptation in humans. Nature Neurosci 1:524-528 
Deubel H (1995) Separate adaptive mechanisms for the control of reactive and volitional saccadic eye movements. Vision Res 35:3529-3540

Deubel H, Wolf W, Hauske G (1986) Adaptive gain control of saccadic eye movements. Hum Neurobiol 5:245-253

Erkelens CJ, Hulleman J (1993) Selective adaptation of internally triggered saccades made to visual targets. Exp Brain Res 93:157-164

Fitzgibbon EJ, Goldberg ME, Segraves MA (1986) Short term saccadic adaptation in the monkey. In: Keller E, Zee DS (eds) Adaptive processes in the visual and oculomotor systems. Pergamon, Oxford, pp 329-333

Fox PT, Fox JM, Raichle ME, Burde RM (1985) The role of cerebral cortex in the generation of voluntary saccades: a positron emission tomographic study. J Neurophysiol 54:348-369

Frens MA, Van Opstal AJ (1994) Transfer of short-term adaptation in human saccadic eye movements. Exp Brain Res 100:293-306

Frens MA, Van Opstal AJ (1997) Monkey superior colliculus activity during short-term saccadic adaptation. Brain Res Bull $43: 473-483$

Friston KJ, Worsley KJ, Frakowiak RSJ, Mazziotta JC (1994) Assessing the significance of focal activations using their spatial extent. Hum Brain Map 1:214-220

Fuchs AF, Robinson FR, Straube A (1993) Role of the caudal fastigial nucleus in saccade generation. I. Neuronal discharge pattern. J Neurophysiol 70:1723-1740

Fuchs AF, Reiner D, Pong M (1996) Transfer of gain changes from targeting to other types of saccade in the monkey: constraints on possible sites of saccadic gain adaptation. J Neurophysiol 76:2522-2535

Fujikado T, Noda H (1987) Saccadic eye movements evoked by microstimulation of lobule VII of the cerebellar vermis of macaque monkeys. J Physiol 394:573-594

Goffart L, Pélisson D (1998) Orienting gaze shifts during muscimol inactivation of caudal fastigial nucleus in the cat. I. Gaze dysmetria. J Neurophysiol 79:1942-1958

Goldberg ME, Musil SY, Fitzgibbon EJ, Smith M, Olson CR (1993) The role of the cerebellum in the control of saccadic eye movements. In: Mano M, Hamada I, DeLong MR (eds) Role of the cerebellum and basal ganglia in voluntary movements. Elsevier, Amsterdam, pp 203-211

Guillaume A, Pélisson D (1999) Saccadic gaze shifts evoked by electrical stimulation of the superior colliculus following caudal fastigial nucleus inactivation in the cat. Soc Neurosci Abstr 25:5

Guitton D (1991) Control of saccadic eye and gaze movements by the superior colliculus and basal ganglia. In: Carpenter RHS (ed) Eye movements. Macmillan Press, Houndmills, pp 244-276

Harris CM (1995) Does saccadic undershoot minimize saccadic flight-time? A Monte-Carlo study. Vision Res 35:691-701

Hashimoto M, Ohtsuka K (1995) Transcranial magnetic stimulation over the posterior cerebellum during visually guided saccades in man. Brain 118:1185-1193

Henson DB (1978) Corrective saccades: effects of altering visual feedback. Vision Res 18:63-67

Jordan MI (1990) Motor learning and the degrees of freedom problem. In: Jeannerod $M$ (ed) Attention and performance XIII: motor representation and control. Erlbaum, Hillsdale, pp 796-836

Jordan MI, Rosenbaum DA (1989) Action. In: Posner MI (ed) Foundations of cognitive science. MIT press, Cambridge, pp 727-768

Kase M, Miller DC, Noda H (1980) Discharges of Purkinje cells and mossy fibers in the cerebellar vermis of the monkey during saccadic eye movements and fixation. J Physiol 300:539-555

Keller EL, Slakey DP, Crandall WF (1983) Microstimulation of the primate cerebellar vermis during saccadic eye movements. Brain Res 288:131-143

Kommerell G, Olivier D, Theopold H (1976) Adaptive programming of phasic and tonic components in saccadic eye movements. Investigations of patients with abducens palsy. Invest Ophthalmol 15:657-660
Kröller J, Pélisson D, Prablanc C (1996) On the short-term adaptation of eye saccades and its transfer to head movements. Exp Brain Res 111:477-482

Law I, Svarer C, Holm S, Paulson OB (1997) The activation pattern in normal humans during suppression, imagination and performance of saccadic eye movements. Acta Physiol Scand 161:419-434

Lee C, Rohrer WH, Sparks DL (1988) Population coding of saccadic eye movements by neurons in the superior colliculus. Nature 332:357-360

Lefèvre P, Quaia C, Optican LM (1998) Distributed model of control of saccades by superior colliculus and cerebellum. Neural Networks 11:1175-1190

Lewis RF, Zee DS (1993) Ocular motor disorders associated with cerebellar lesions: pathophysiology and topical localization. Rev Neurol (Paris) 149:665-677

Lynch JC (1992) Saccade initiation and latency deficits after combined lesions of the frontal and posterior eye fields in monkeys. J Neurophysiol 68:1913-1916

Matin L (1982) Visual location and eye movements. In: Wertheim AH, Wagenaar AW, Leibowitz HW (eds) Tutorials on motion perception. Plenum Press, New York, pp 101-156

May PJ, Hartwich-Young R, Nelson J, Sparks DL, Porter JD (1990) Cerebellotectal pathways in the macaque: implications for collicular generation of saccades. Neuroscience 36:305-324

McLaughlin SC (1967) Parametric adjustment in saccadic eye movement. Percept Psychophysiol 2:359-362

Melis BJ, Gisbergen JA van (1996) Short-term adaptation of electrically induced saccades in monkey superior colliculus. J Neurophysiol 76:1744-1758

Miller JM, Anstis T, Templeton WB (1981) Saccadic plasticity: parametric adaptive control by retinal feedback. J Exp Psychol Hum Percept Perform 7:356-366

Neter J, Wasserman W, Kutner MH (1990) Applied linear models. Irwin, Boston

Noda H (1991) Cerebellar control of saccadic eye movements: it neural mechanisms and pathways. Jpn J Physiol 41:351-368

Noda H, Fujikado T (1987) Topography of the oculomotor area of the cerebellar vermis in macaques as determined by microstimulation. J Neurophysiol 58:359-378

Noda H, Sugita S, Ikeda Y (1990) Afferent and efferent connections of the oculomotor region of the fastigial nucleus in the macaque monkey. J Comp Neurol 302:330-348

Noto CT, Watanabe S, Fuchs AF (1999) Characteristics of simian adaptation fields produced by behavioral changes in saccade size and direction. J Neurophysiol 81:2798-2813

O'Sullivan EP, Jenkins IH, Henderson L, Kennard C, Brooks DJ (1995) The functional anatomy of remembered saccades: a PET study. Neuroreport 6:2141-2144

Ohtsuka K, Noda H (1991a) The effect of microstimulation of the oculomotor vermis on discharges of fastigial neurons and visually directed saccades in macaques. Neurosci Res 10:290-295

Ohtsuka K, Noda H (1991b) Saccadic burst neurons in the oculomotor region of the fastigial nucleus of macaque monkeys. J Neurophysiol 65:1422-1434

Ohtsuka K, Noda H (1995) Discharge properties of Purkinje cells in the oculomotor vermis during visually guided saccades in the macaque monkey. J Neurophysiol 74:1828-1840

Optican LM (1982) Saccadic dysmetria. In: Lennerstrand G, Zee DS, Keller EL (eds) Functional basis of ocular motility disorders. Pergamon, Headington Hill Hall, pp 441-451

Optican LM, Robinson DA (1980) Cerebellar-dependent adaptive control of primate saccadic system. J Neurophysiol 44:10581076

Optican LM, Zee DS, Chu FC (1985) Adaptive response to ocular muscle weakness in human pursuit and saccadic eye movements. J Neurophysiol 54:110-122

Paus T (1996) Location and function of the human frontal eyefield: a selective review. Neuropsychologia 34:475-483

Paus T, Petrides M, Evans AC, Meyer E (1993) Role of the human anterior cingulate cortex in the control of oculomotor, manual, and speech responses: a positron emission tomography study. J Neurophysiol 70:453-469 
Paus T, Marrett S, Worsley KJ, Evans AC (1995) Extraretinal modulation of cerebral blood flow in the human visual cortex: implications for saccadic suppression. J Neurophysiol 74:2179-2183

Pélisson D, Prablanc C, Urquizar C (1988) Vestibuloocular reflex inhibition and gaze saccade control characteristics eye-head orientation in humans. J Neurophysiol 59:997-1013

Petit L, Orssaud C, Tzourio N, Salamon G, Mazoyer B, Berthoz A (1993) PET study of voluntary saccadic eye movements in humans: basal ganglia-thalamocortical system and cingulate cortex involvement. J Neurophysiol 69:1009-1017

Petit L, Orssaud C, Tzourio N, Crivello F, Berthoz A, Mazoyer B (1996) Functional anatomy of a prelearned sequence of horizontal saccades in humans. J Neurosci 16:3714-3726

Phillips JO, Fuchs AF, Ling L, Iwamoto Y, Votaw S (1997) Gain adaptation of eye and head movement components of simian gaze shifts. J Neurophysiol 78:2817-2821

Pierrot-Deseilligny C, Rivaud S, Gaymard B, Agid Y (1991) Cortical control of reflexive visually guided saccades. Brain 114:1473-1485

Pierrot-Deseilligny C, Rivaud S, Gaymard B, Muri R, Vermersch AI (1995) Cortical control of saccades. Ann Neurol 37:557567

Prablanc C, Jeannerod M (1975) Corrective saccades: dependence on retinal reafferent signals. Vision Res 15:465-469

Redding GM, Wallace B (1996) Adaptative spatial alignment and strategic perceptual-motor control. J Exp Psychol Hum Percept Perform 22:379-394

Rivaud S, Muri RM, Gaymard B, Vermersch AI, PierrotDeseilligny C (1994) Eye movement disorders after frontal eye field lesions in humans. Exp Brain Res 102:110-120

Robinson DA (1973) Models of the saccadic eye movements control system. Kybernetic 14:71-83

Robinson FR, Straube A, Fuchs AF (1993) Role of the caudal fastigial nucleus in saccade generation. II. Effects of muscimol inactivation. J Neurophysiol 70:1741-1758

Ron S, Robinson DA (1973) Eye movement evoked by cerebellar stimulation in the alert monkey. J Neurophysiol 36:1004-1022

Rosen BR, Buckner RL, Dale AM (1998) Event-related functional MRI: past, present, and future. Proc Natl Acad Sci USA 95:773-780

Rossetti Y (1994) De la main vers son but: integrations visuo-proprioceptives dans l'elaboration du mouvement. Universite Claude Bernard, Lyon

Rossetti Y, Koga K, Mano T (1993) Transient change in eye-hand coordination during early exposure to wedge-prism. Percept Psychophysiol 54:355-364

Sato H, Noda H (1992) Saccadic dysmetria induced by transient functional decortication of the cerebellar vermis. Exp Brain Res 88:455-458

Schall JD (1991) Neuronal activity related to visually guided saccades in the frontal eye fields of rhesus monkeys: comparison with supplementary eye fields. J Neurophysiol 66:559-579

Schiller PH, True SD, Conway JL (1980) Deficits in eye movements following frontal eye-field and superior colliculus ablations. J Neurophysiol 44:1175-1189
Schweighofer N, Arbib MA, Dominey PF (1996) A model of the cerebellum in adaptive control of saccadic gain. I. The model and its biological substrate. Biol Cybern 75:19-28

Scudder CA, Batourina EY, Tunder GS (1998) Comparison of two methods of producing adaptation of saccade size and implications for the site of plasticity. J Neurophysiol 79:704-715

Sommer MA, Tehovnik EJ (1997) Reversible inactivation of macaque frontal eye field. Exp Brain Res 116:229-249

Sparks DL (1986) Translation of sensory signals into commands for control of saccadic eye movements: role of primate superior colliculus. Physiol Rev 66:118-171

Sparks DL, May LE (1990) Signal transformations required for the generation of saccadic eye movements. Annu Rev Neurosci 13:309-336

Straube A, Deubel H, Spuler A, Buttner U (1995) Differential effect of a deep bilateral cerebellar nuclei lesion on externally and internally triggered saccades. Neuroophtalmology 15:6174

Straube A, Fuchs AF, Usher S, Robinson FR (1997) Characteristics of saccadic gain adaptation in rhesus macaques. J Neurophysiol 77:874-895

Sweeney JA, Mintun MA, Kwee S, Wiseman MB, Brown DL, Rosenberg DR, Carl JR (1996) Positron emission tomography study of voluntary saccadic eye movements and spatial working memory. J Neurophysiol 75:454-468

Takagi M, Zee DS, Tamargo RJ (1998) Effects of lesions of the oculomotor vermis on eye movements in primate: saccades. J Neurophysiol 80:1911-1931

Talairach J, Tournoux P (1988) Co-planar stereotaxic atlas of the human brain. Thieme, Stuttgart

Waespe W, Baumgartner R (1992) Enduring dysmetria and impaired gain adaptativity of saccadic eye movements in Wallenberg's lateral medullary syndrome. Brain 115:1125-1146

Wallman J, Fuchs AF (1998) Saccadic gain modification: visual error drives motor adaptation. J Neurophysiol 80:2405-2416

Winstein CJ, Grafton ST, Pohl P (1997) Motor task difficulty and brain activity: investigation of goal directed reciprocal aiming using positron emission tomography. J Neurophysiol 77:15811594

Woods RP, Iacobini M, Grafton ST, Mazziotta JC (1996) Threeway analysis of variance. In: Myers R, Cunningham V, Bailey D (eds) Quantification of brain function using PET. Academic Press, New York, pp 353-358

Woods RP, Grafton ST, Holmes CJ, Cherry SR, Mazziotta JC (1998a) Automated image registration. 1. General methods and intra-subject validation. J Comput Assist Tomogr 22:139-152

Woods RP, Grafton ST, Watson JDG, Sicotte NL, Mazziotta JC (1998b) Automated image registration. 2. Intersubject validation of linear and nonlinear models. J Comput Assist Tomogr 22: $153-165$

Yamada J, Noda H (1987) Afferent and efferent connections of the oculomotor cerebellar vermis in the macaque monkey. J Comp Neurol 265:224-241

Zee DS, Yee RD, Cogan DG, Robinson DA, Engel WK (1976) Ocular motor abnormalities in hereditary cerebellar ataxia. Brain 99:207-234 